\title{
Characterization of a polarimetric infrared imager based on the orthogonality breaking technique
}

\author{
François Parnet $^{1}{ }^{*}$, Julien Fade ${ }^{1}$, Muriel Roche $^{2}$, Goulc'hen Loas ${ }^{1}$, Noé Ortega-Quijano ${ }^{1,{ }^{\dagger}}$, \\ Ludovic Frein $^{1}$, Mehdi Alouini ${ }^{1}$ \\ ${ }^{1}$ Univ Rennes, CNRS, Institut FOTON - UMR 6082, F-35000 Rennes, France \\ ${ }^{2}$ Aix Marseille Univ, CNRS, Centrale Marseille, Institut Fresnel, Marseille, France
}

\begin{abstract}
This paper reports an in-depth experimental characterization and analysis of an infrared active polarimetric imaging system based on the orthogonality breaking polarization-sensing approach. We first recall the principle of this laser scanning polarimetric imaging technique, based on the illumination of a scene by means of a dual-frequency dual-polarization light source. The experimental design is then described, along with measurements on test scenes with known polarimetric properties used to validate/calibrate the imaging system, and to characterize its optical properties (sensitivity, resolution). The noise sources that temporally and spatially affect the quality of the orthogonality breaking data are then investigated. Our results show that the raw temporal signals detected at a given location of the scene are perturbed by Gaussian fluctuations, while the spatial information contained in the images acquired through raster scan of the scene are dominated by speckle noise, which is a common characteristic of active polarimetric imaging systems. Finally, the influence of the source temporal coherence on the images is analyzed experimentally, showing that orthogonality breaking acquisitions can still be performed efficiently with a low-coherence source.
\end{abstract}

Keywords: active imaging, polarimetric imaging, instrument design, infrared imaging, microwave photonics.

*Corresponding author, francois.parnet@univ-rennes1.fr

$\dagger$ Now at Deneb Medical, S.L., San Sebastian, Spain

\section{Introduction}

Active polarimetry is a useful tool to enhance the detection capabilities of standard intensity imagers. Indeed, polarization contrasts can highlight regions of interest with similar reflectance, but which exhibit different polarization behavior. ${ }^{1-5}$ This technique stands on the illumination of a scene with a polarized source and the detection of the backscattered light through a polarizing filter, conventionally named analyzer. Exploiting different states of polarization at the emission and the analysis of light may lead to the characterization of the complete polarimetric response of the scene, for instance using the 16-acquisitions Mueller imaging technique. The number of measurements can be reduced to shorten the acquisition time while addressing specific parameters of the scene polarimetric response or, at least, to display relevant contrasts. ${ }^{6}$ For instance, the orthogo- 
nal states contrast (OSC) technique consists in emitting a linearly polarized source and acquiring two images through parallel and crossed polarizers at the analysis of light, in order to provide an enhanced contrast image..$^{7,8}$

In the context of target detection applications, near infrared (NIR) active polarimetric imaging can advantageously contribute to reveal manufactured objects hidden in a natural environment. ${ }^{8-10}$ In particular, NIR operation at $\lambda=\mathbf{1 . 5 5} \mu \mathrm{m}$ is suited for such an application since it considerably reduces the ocular hazard ${ }^{11,12}$ while ensuring a relatively stealth active imager. However, performing such an imaging modality simultaneously at high speed (video frame rate) to monitor dynamic scenarios, and at long range (several hundreds of meters) still remains a challenge. In this context, we recently reported the development of an in-lab polarimetric imaging demonstrator at $1.55 \mu \mathrm{m}$ that relies on the depolarization/dichroism sensing by orthogonality breaking (DSOB) technique to yield contrasts on a scene through a single acquisition that lasts approximately 1 s. ${ }^{13}$ As stated in Ref. 13, such an imaging system can be of interest for practical implementation in defence applications: the optical power deposited on the scene is maximized, measurements can be performed at high speed, and the polarimetric information consistency is preserved. Moreover, the DSOB technique can provide simultaneously two relevant images (contrast and phase) while the OSC technique results in a single contrast image. However, the characterization of such a demonstrator (response of the detection stage, noise affecting the signals) is still pending.

The DSOB technique is an unconventional approach which employs a dual-frequency dualpolarization (DFDP) light source to enlighten the scene, and after interaction with it, the polarization information is encoded in a radiofrequency (RF) beatnote signal that is retrieved through heterodyne detection by a high speed photodetector. ${ }^{14}$ A DFDP source comprises two electro- 
magnetic fields orthogonally polarized and shifted in frequency with a detuning, noted $\Delta \nu$, lying within the RF range. When interacting with a non dichroic and non depolarizing sample, both states of polarization of the light source may change due to birefringence effects, however they remain orthogonal and the detected intensity is constant in time. This property makes the DSOB technique a good candidate to polarimetric endoscopy applications. ${ }^{14-16}$ However, if the sample introduces dichroism, each state of polarization is partially projected onto the other one, thus resulting in the breaking of the polarimetric orthogonality. As a result, the fields interfere, which produces a modulation of the signal at the frequency detuning $\Delta \nu$. In the specific case where the illumination beam is circularly left/right polarized, the beatnote signal leads to the characterization of the sample's diattenuation ratio and its orientation in a straightforwardly and independent fashion. ${ }^{17}$ This property will be specifically addressed in this article, even though we reported in previous works ${ }^{14,15}$ the sensing of depolarization effects with the DSOB technique. This can occur when the experimental conditions imply partial averaging over only a few spatial modes of the field. ${ }^{15}$ Such conditions will clearly not be fulfilled in the experimental context of this article addressing NIR active polarimetric imaging, and as a result the DSOB technique will not be sensitive to pure depolarization in the present case.

In our previous work, a NIR demonstrator design of a DSOB imaging system was reported and experimental results on a synthetic scene were compared with a conventional OSC imager. ${ }^{13}$ The present article is dedicated to the study of this unconventional imager with a thorough characterization of its spatial resolution (or instantaneous field-of-view) as well as the noise affecting the images. Such an experimental study should contribute to determine the limits (physical, technical) of the demonstrator and should pave the way to future experiments.

After a brief description of the DSOB imager design in Sec. 2, the analysis of the detection 
stage and the noises which dominate it are tackled in Sec. 3. Section. 4 is dedicated to the analysis of the imaging system. Furthermore, since the measurements are directly linked to the properties of the DFDP source ${ }^{18}$ (states of polarization, fields power balance, ...), Sec. 5 presents an insight into the impact of the source spectral properties on the resulting polarimetric images. Lastly, conclusions and outlooks are discussed in Sec. 6.

\section{Imaging system design}

The imaging system comprises three main building blocks used to: (A) produce the DFDP light beam at $1.55 \mu \mathrm{m}$, (B) enlighten the scene and, (C) detect the backscattered light and build the images. Figure 1 depicts the imager scheme. The content and principle of each block are detailed in the following subsections.

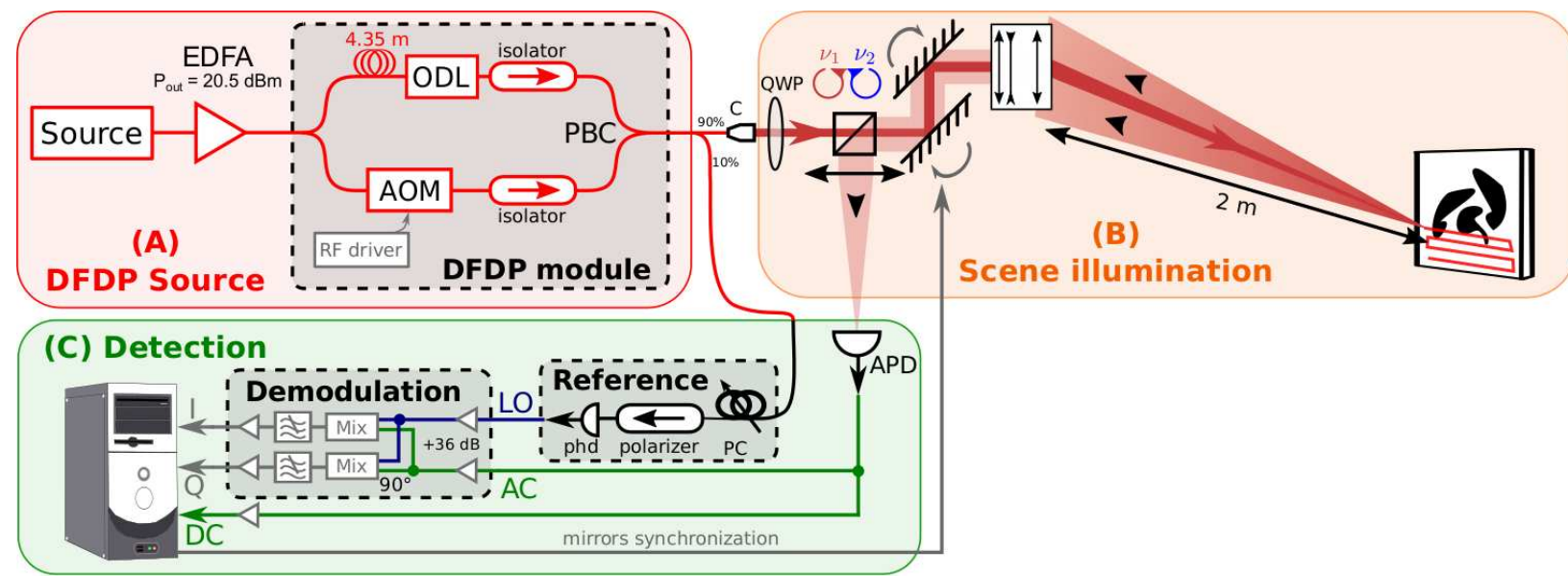

Fig 1 Scheme of the NIR DSOB imaging system. Refer to text in Sec. 2 for details.

\subsection{DFDP source - Block A}

Firstly, a coherent laser (Yenista, Tunics 100HP-CL), tunable on C-L telecom bands (from $1500 \mathrm{~nm}$ to $1630 \mathrm{~nm}$ ), is amplified by a two stage polarizing maintaining (PM) erbium-doped fiber amplifier 
(EDFA) such that the maximum output optical power reaches $112 \mathrm{~mW}(20.5 \mathrm{dBm})$. The amplified source then enters an all-fibered module (comprising only PM components) that produces a DFDP beam at $\lambda=1.55 \mu \mathrm{m} .{ }^{13}$ It is a Mach-Zehnder interferometer-like module (noted DFDP module in Fig. 1) which splits the incoming light into two arms, introduces a frequency detuning $\Delta \nu=80 \mathrm{MHz}$ with an acousto-optic modulator (AOM) in one arm, and mixes both light beams with orthogonal linear states of polarization in a polarization beam combiner (noted PBC). It must be noted that the optical power actually deposited on the scene is about $10 \mathrm{~mW}$, due to the losses induced by the couplers, the AOM and the free-space optics (beamsplitter). In an optimized version of the system, the optical losses could be significantly reduced by using appropriately chosen custom components instead of off-the-shelf devices inside the DFDP module and/or by replacing the free-space beamsplitter with a hole mirror.

As detailed in Ref. 13, balancing the fiber length of the arms of this Mach-Zehnder interferometerlike module is essential to detect the modulated signal. Indeed, it prevents the phase noise of the front laser from affecting the demodulation process. The accuracy required to balance the arms length is related firstly to the temporal coherence of the front source, and secondly, to the phase adjustment of both optical waves exiting the fibered module. For instance, it can be estimated that the highly coherent Tunics laser requires a maximum arms length mismatch of just a few centimeters. In Sec. 5.2, two other sources that feature temporal coherence lower than the Tunics laser's will be used to explore the influence of the source temporal coherence on the measurements and will thus require a much better accuracy. To accurately balance the arms length of the DFDP module, a tunable PM optical delay line (ODL) has been introduced in the arm without the AOM component. For these sources, the balance accuracy has been estimated to be of a few tens of micrometers (see Sec. 5.2). An accurate balancing of the arms length is performed thus enabling a 
correct detection of the signals with all three sources.

\subsection{Scene illumination - Block B}

A PM splitter sends $90 \%$ of the DFDP source power in free space through a collimation lens (Newport, F-H10-IR-APC). A removable quarter waveplate, whose eigenaxes are oriented at $45^{\circ}$ from the DFDP source axes, allows the linear states of polarization to be turned into circular ones, at will. The DFDP beam is then transmitted through a 50:50 polarization insensitive beamsplitter used to separate the forward and backward optical paths of light. A pair of galvanometric mirrors $(6220 \mathrm{H}$, Cambridge Technology) scans the beam over the scene. Although the field-of-view (FOV) of the imager can be tuned by changing the amplitude of the mirror scan, the FOV will be defined to $45 \operatorname{mrad}\left(2.6^{\circ}\right)$ in all the experiments reported in this article. Such a low FOV ensures low residual changes of the polarization states of the DFDP source (upon reflection on the mirrors), as well as it preserves the Gaussian shape of the beam focused on the scene and ensures constant efficiency of the backscattered light collection through the beam-forming optics. The beam is focused at a distance of two meters from the source by a Cooke's triplet lens arrangement, which comprises three 2-inch diameter lenses, near infrared (NIR) anti-reflection (AR) coated. This lens arrangement provides a $\pm 10 \mathrm{~cm}$ depth of focus and reduces the optical aberrations as well, ensuring that the focused spot has a constant shape across all the object plane. The laser scanning operation enables maximum power deposit at each location of the scene which is an asset for long range active imaging applications. 


\subsection{Light detection and image building - Block C}

The light coming from the scene is collected through the Cooke's triplet, the galvanometric mirrors and the beamsplitter. A $50 \mathrm{~mm}$ focal lens (NIR AR coated) focuses the collected light on a $200 \mu \mathrm{m}$ diameter (active area) avalanche photodiode (APD) with a $100 \mathrm{MHz}$ bandwidth integrated transimpedance amplifier (PLA-641, Princeton Lightwave). Initially dedicated to the detection of signals in differential mode, the APD comprises two outputs, which we use to retrieve separately the mean (DC) and the modulated (AC) components from the detected signal. The AC channel (DC-filtered) reaches a theoretical sensitivity of $170 \mathrm{mV} / \mu \mathrm{W}$ (manufacturer data). The DC channel (AC-filtered) is amplified by a low noise low frequency amplifier for an overall theoretical sensitivity of $\mathbf{4 0 8 0} \mathrm{mV} / \mu \mathrm{W}$. Diverting the differential operation mode of the APD leads to an offset on the DC channel that is compensated with the low noise amplifier. However, the DC channel dark current remains above $290 \mathrm{mV}$ and was observed to fluctuate slightly $( \pm 20 \mathrm{mV})$ at a $10 \mathrm{~ms}$ timescale. It can be noted that the detection of light is performed with a free space photodetector though it could be done with a fibered one. The chosen free space configuration ensures ease of control of the polarization at the emission and the collection of light, independently.

The high speed modulation $(80 \mathrm{MHz})$ of the $\mathrm{AC}$ channel is frequency-transposed into two continuous components by a homemade synchronous demodulation device that enables real time acquisition of the amplitude and the phase of the AC signal with an I/O card. To demodulate the high frequency beatnote, a reference signal at $\Delta \nu=80 \mathrm{MHz}$ is required as local oscillator (LO). It is produced by a PM splitter which guides $10 \%$ of the DFDP beam to a polarization controller (PC) followed by an in-line polarizer and a fibered InGaAs photodiode. The PC enables control of the source polarization projection onto the polarizer. Thus, the RF power of the LO signal can be 
tailored and, in our case, set to $-26 \mathrm{dBm}$, in order to match (after amplification and splitting, see below) the $+7 \mathrm{dBm}$ nominal operating point of the mixers for maximum linearity.

In the demodulation circuit, both $\mathrm{AC}$ and LO input signals are amplified (36 dB gain, ZX60100VH+, Minicircuits) and splitted in two copies (3 dB losses) by power splitters, Minicircuits, ZFRSC-42+ for AC and ZMSCQ-2-90 for LO, the latter component introducing a $\pi / 2$ phase shift on one copy of the LO signal. Both AC signals are multiplied witch each LO copy using two mixers (Minicircuits, ZLW-6+) to generate the In-phase (I) and Quadrature (Q) components. These signals are frequency cleaned by a $5^{\text {th }}$ order low pass Butterworth filter (frequency cutoff $f_{c}=250 \mathrm{kHz}$ ) and are amplified by $20 \mathrm{~dB}$ to take advantage of the 16-bits analog-to-digital converter (ADC) module of the I/O card (USB-6356, National Instruments) used in a $\pm 5 \mathrm{~V}$ input range. While DC, I and Q signals are digitally converted, the I/O card digital-to-analog converter (DAC) module produces two electrical signals to set the galvanometric mirrors orientation, and hence the beam position on the scene. A LabVIEW (National Instruments) code drives the synchronization between the mirrors orientation and the signal acquisition to associate each acquired value with a location on the scene during raster scanning. The DC, I and Q raw images are built with $\mathrm{N}$ pixels side (square images) where the value $\mathrm{N}$ is set by the user who may also choose the FOV and the acquisition rate (limited by the mirrors speed). From such data, the beatnote amplitude $(|\mathrm{AC}|)$, the phase $(\phi)$ and the orthogonality breaking contrast (OBC) images can be derived, displayed and recorded with 
following definitions: ${ }^{13}$

$$
\begin{aligned}
|\mathrm{AC}| & =\sqrt{\mathrm{I}^{2}+\mathrm{Q}^{2}}, \\
\phi & =\operatorname{atan}(\mathrm{Q} / \mathrm{I}), \\
\mathrm{OBC} & =|\mathrm{AC}| / \mathrm{DC} .
\end{aligned}
$$

Further mathematical details can be found in Ref. 15 which extensively describes how the phase $(\phi)$ and the $\mathrm{OBC}$ calculated parameters can be related to the linear diattenuation properties of samples.

\section{Characterization of the detection stage}

As described above, an orthogonality breaking imaging system uses a detection stage which is unconventional for imaging applications and that justifies the analysis of its impact on the acquired signals. For this purpose, the response of the detection chain (from the photodetector to the I/O card) is investigated along with the noise distribution of the digitized signals.

\subsection{Avalanche photodiode response}

The photodiode response is evaluated experimentally by measuring the DC signal and the amplitude of the AC signal while the power of the DFDP beam collected by the APD is varied. The 50:50 beamsplitter has been replaced by a mirror to steer the collimated beam on the APD. A polarizer (POL-NIRA-050, Thorlabs) has been inserted in front of the detector to produce a maximum beatnote and characterize simultaneously the DC and AC responses. Both DC and AC outputs of the APD module were found to be operating in a roughly linear regime with experimental sensitivities 
of $3550 \mathrm{mV} / \mu \mathrm{W}$ and $155 \mathrm{mV} / \mu \mathrm{W}$ respectively for input powers lying between 0.1 and $1 \mu \mathrm{W}$. Outside this range, the APD starts exhibiting a non-linear behavior, and reliable acquisition of physical measurements would require compensating such non linear response from calibration.

\subsection{Demodulation response}

As the I/O card is not able to directly sample the AC signal delivered by the APD, the signal is frequency down-converted through the demodulation system. Two continuous components, I and $\mathrm{Q}$, are then produced and digitized along with the DC signal. The APD response affects both DC and AC signals while the demodulation response impacts only the latter. The demodulation response is measured by varying the amplitude of the AC input signal while the amplitude of the LO reference signal is kept constant. A two-channel function generator (AFG3252C, Tektronix) is used to deliver controlled AC and LO signals modulated at $80 \mathrm{MHz}$ to the demodulation board. The RF power of the LO signal is set to $-26 \mathrm{dBm}$. The demodulation response is measured while the amplitude of the input AC signal is varied between 1 to $100 \mathrm{mV}$. From 1 to $25 \mathrm{mV}$, the demodulation exhibits a linear response such that there is a voltage gain of $40 \mathrm{~dB}$ between the estimated $|\mathrm{AC}|$ amplitude derived from Eq. 1 and the RF input amplitude. Beyond $25 \mathrm{mV}$, the demodulation saturates and the gain decreases. The phase, which can be derived from Eq. 2, remains fairly constant while the beatnote amplitude is varied.

The demodulation board will be used on the whole range (from 1 to $100 \mathrm{mV}$ ) though it exhibits a non linear behavior above $25 \mathrm{~mW}$. Indeed, if only qualitative polarimetric contrasts are sought, the saturation does not compromise the differentiation of low and strong modulations. On the other hand, if quantitative contrasts are required to extract physical quantities (e.g. diattenuation coefficient), a non linear calibration of the overall detection stage can be realized. Regarding the 
imaging setup, a simple linear calibration is detailed for weak input DC $(<1 \mathrm{~V})$ and AC signals $(<25 \mathrm{mV})$ in Sec. 4.3 .

\subsection{Temporal noise}

The noise of the three raw signals (DC, I and Q) is studied temporally while the galvanometric mirrors orientation remains static. The circularly (left/right) polarized DFDP beam is focused and reflected on a metallic mirror placed at a distance of $2 \mathrm{~m}$. A NIR polarizer is placed in front of the detector and oriented so as to produce a beatnote signal allowing us to characterize the noise that affects all three raw signals.

During the development of the imaging setup, first temporal acquisitions on this scene exhibited spurious peaks on the signals. Figure 2(a) illustrates the presence of multiple frequencies in the spectrum of the component $\mathrm{Q}$, acquired during $1 \mathrm{~s}$. To reduce the presence of these undesired frequencies, the low-pass filters frequency cutoff has been optimized to $250 \mathrm{kHz}$, a noisy power supply was replaced with a low noise linear supply and high power electronic cards (driving the galvanometric mirrors position) were electromagnetically shielded. Figure 2(b) shows the Q component spectrum after this optimization step showing strong reduction of the spurious noise peaks.

The nature of the noise is identified by plotting the distributions of the signals measured while the DFDP beam is focused on the polarizer at $2 \mathrm{~m}$. DC, I and Q signals are acquired during $1 \mathrm{~s}$ with 1 million points. Prior to the signals acquisition, the APD dark current has been measured for all three signals and subtracted to the measurements (on the polarizer). Histograms of the three raw signals, displayed in Fig. 3 (a-c), are fitted with Gaussian distributions (continuous black line) evidencing the fact that temporal noise is Gaussian with this optimized detection setup. Since 


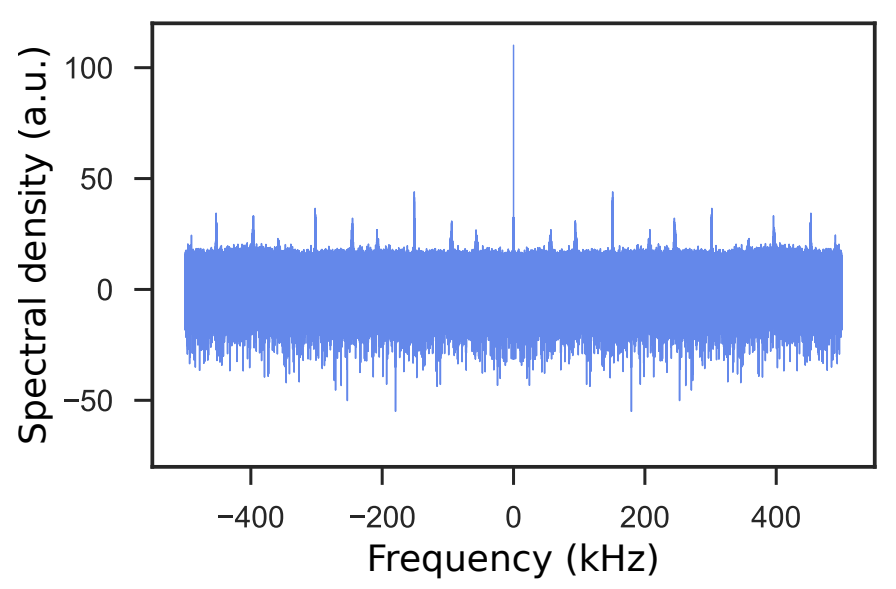

(a)

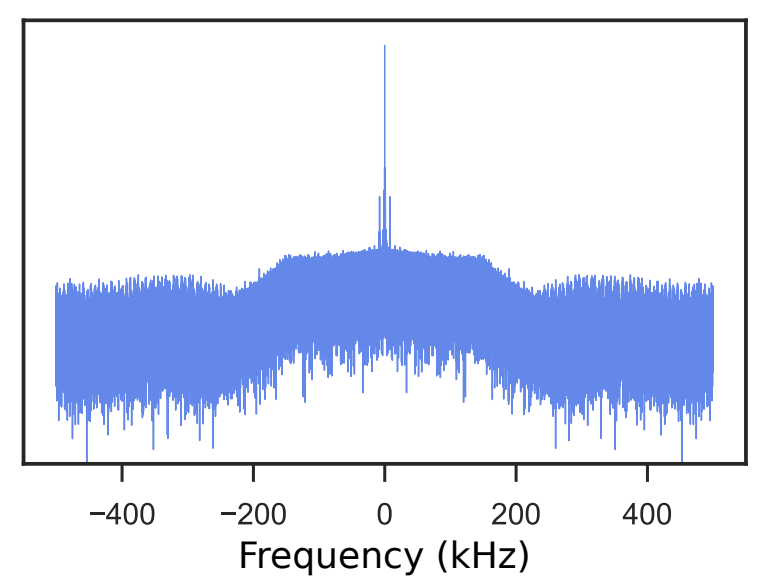

(b)

Fig 2 Q quadrature component spectra before (a) and after (b) reduction of the noise sources of the demonstrator initial development.
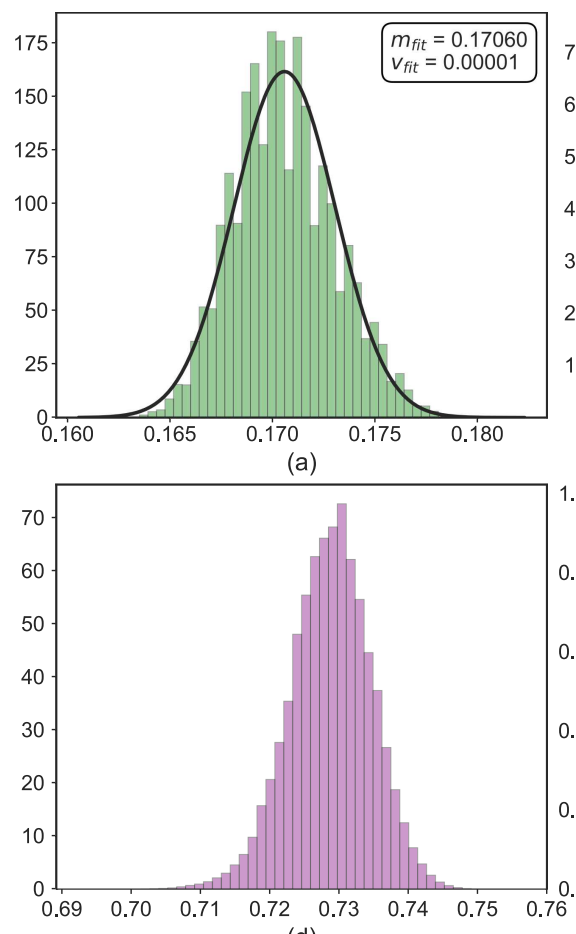

(d)
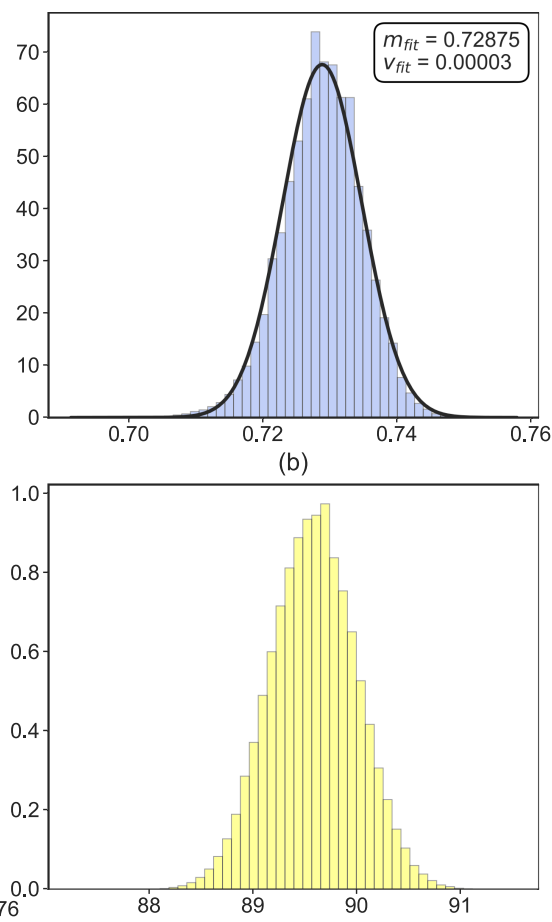

(e)

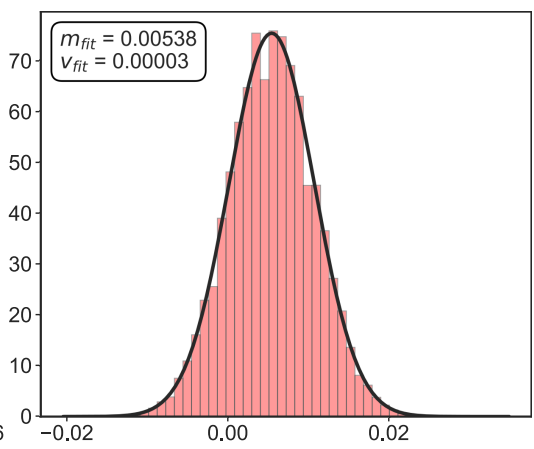

(c)

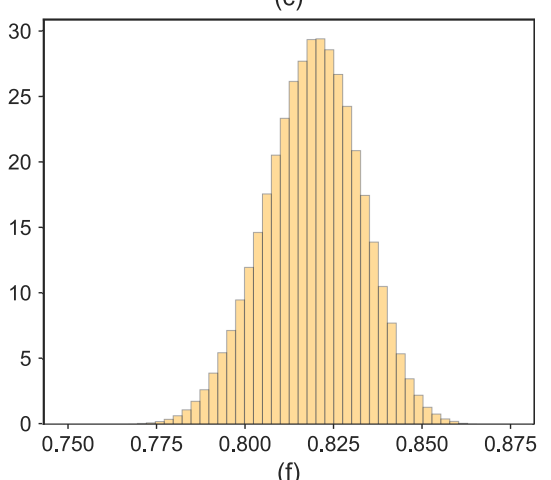

Fig 3 Histograms (50 bins) of the DC (a), I (b), Q (c) raw signals (in volt) and the $|\mathrm{AC}|$ (d) (in volt), $\phi$ (e) (in degrees), OBC (f) calculated signals. Gaussian distributions (black continuous line) are fitted with the raw signals. The mean $\left(m_{f i t}\right)$ and variance $\left(v_{f i t}\right)$ values of each fit are given in the inset of the corresponding graph.

I and Q components are dominated by Gaussian noise, the estimated $|\mathrm{AC}|$ and $\phi$ signals can be described respectively by a Rice distribution and by a specific distribution for the phase, given in Ref. 19, as long as I and Q random variables can be considered as independent and exhibit 
equal variances. However, the expression for the $\mathrm{OBC}$ distribution, derived as the ratio of a Rice distribution over a Gaussian one, could not be determined analytically. In addition, such result has not been referenced yet in the literature, to our best knowledge. Histograms of the signals $|\mathrm{AC}|, \phi$ and OBC are displayed respectively in Fig. 3 (d), (e) and (f).

Here, the variances of the I and Q distributions are equal and constant along changes of their mean value (in the linear range of the response of the detection stage). Beyond the linear range, the independence and the equal variances hypotheses are no longer true and the $|\mathrm{AC}|$ and $\phi$ signals could not be fitted anymore with simple known distributions.

\section{Images characterization}

The previous analysis described the temporal noise distribution of the signals acquired over time while the beam-steering galvanometric mirrors were kept static. In the following, we consider that the mirrors are in motion so that a set of images (DC, I, Q) is built by the imaging system in a single laser scan.

\subsection{APD dark current}

As a first approach, a single scan is performed without light. For this purpose, the active area of the APD is covered and the light source is turned off. It can be noted that this case consists in building images from the dark signals delivered by the detection stage (photodiode and demodulation). Figure 4(a) depicts the corresponding DC, I and Q images.

In the DC image, horizontal lines irregularly distributed can be observed with voltage variations of about $20 \mathrm{mV}$. This spurious noise is due to the temporal slow fluctuations of the APD dark current that causes such spatial pattern during raster scanning of the scene. This effect could be 
reduced by subtracting a statistical value (e.g. mean, median, ...) of the APD dark current to each corresponding line in the images. However, this process would require to sacrifice few pixel columns on the left/right edges of the images. Another possible solution could stand on using a different APD module without this defect. In the present work, no method has been used to reduce this spurious noise.

(a)
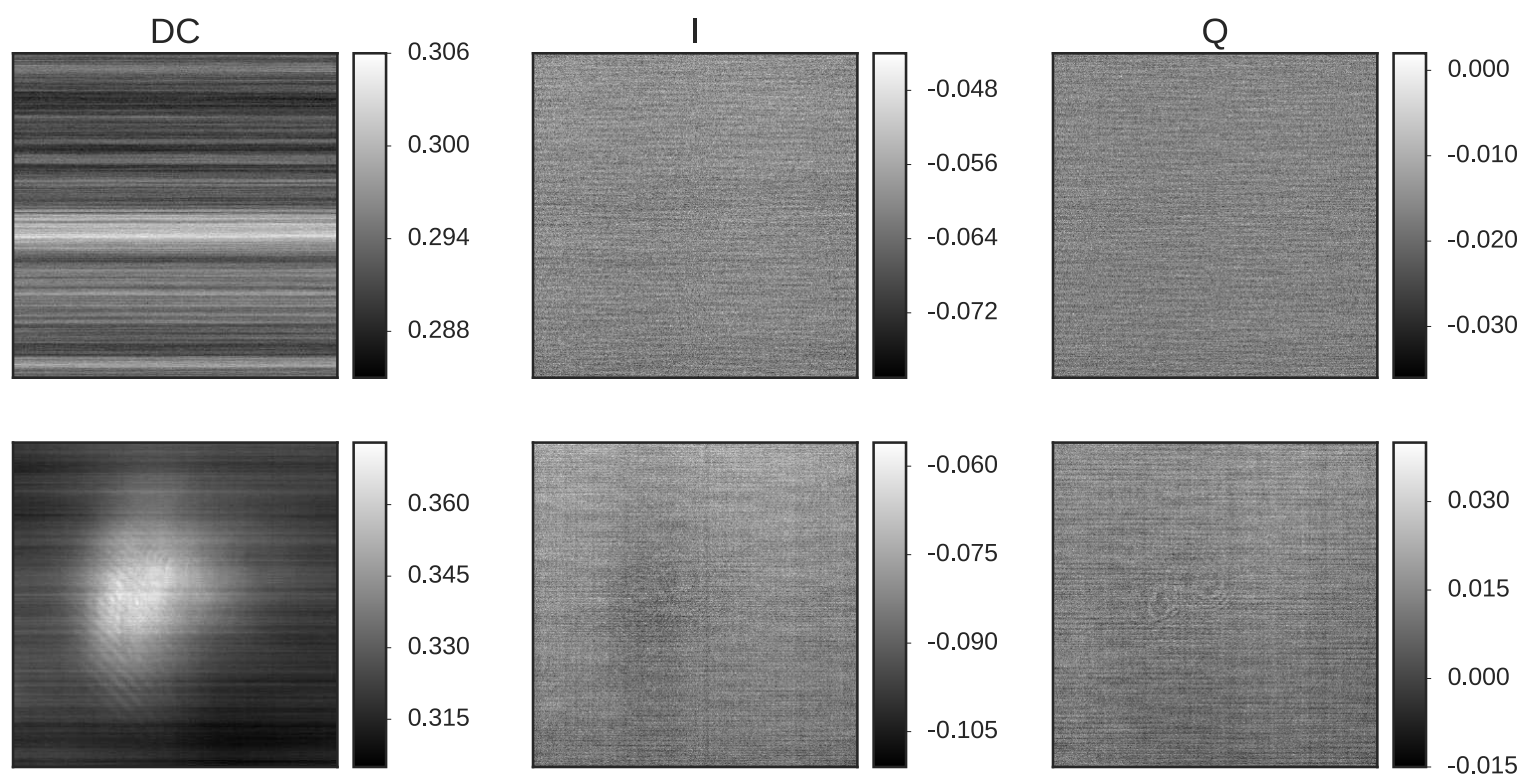

Fig 4 (a) APD dark current raw images. (b) Detection of parasite light from the reflection of the DFDP beam on the beam-forming optics lenses in the absence of backscattered light from the scene.

\subsection{Spurious reflections}

We now consider the case where the light beam is directed towards a scene and the detector receives an insufficient quantity of backscattered light to get an image of it. The raw images displayed in Fig. 4(b) correspond to this situation, which was obtained by using the remote laboratory wall as a distant and low reflectance target. A low quantity of light (approximately $10 \mathrm{nW}$ ) is nevertheless received by the detector and forms a large spot on the DC image. This spurious light arises from the Fresnel reflections of the incident beam on the beam-forming optics even though the lenses 
are coated in the near infrared range $(0.09 \%$ reflection coefficient at $\lambda=1.55 \mu \mathrm{m})$. It can be noticed that these reflections do not appear in the I and Q images as the lenses do not affect the orthogonality of the reflected DFDP beam when the angle of incidence is small. To get rid of these reflections in the DC image, the beam-forming optics is slightly misaligned to translate these reflections outside the field of view of the acquisition. As a consequence, the aberrations reduction provided by the lenses in a Cooke's triplet configuration is slightly degraded. In an optimized system, these spurious reflections could be suppressed by spatial filtering, e.g. by inserting a mask in the Fourier plane .

\subsection{Validation of the demonstrator}

It is of utmost importance to assess the fair agreement between theoretical and experimental results. Thus, a test scenario, encompassing elements with known polarimetric properties, was developed to validate the imaging setup results. The test scene is depicted in Fig. 5(a) and comprises a nondepolarizing metallic plate (labelled as (0) in Fig. 5(a)) with multiple elements above it. Three NIR Polaroids with a dichroic behavior, denoted by (1a) to (1c), are respectively oriented at approximately $0^{\circ}, 90^{\circ}$ and $-45^{\circ}$. Three birefringent elements: two visible Polaroids (which are fairly good polarizers in the visible, but were measured to be almost only birefringent in the NIR range), (2a) and (2b), and an adhesive tape, (3), are placed on the plate next to two depolarizing elements: a black Canson paper, (4), and a sandpaper, (5). The background around the metallic plate is a wooden board, (6), with a depolarizing behavior. It must be noted that the scene is perpendicularly oriented to the illumination so that most of the backscattered light can be collected by the optics. Moreover, this study is limited to flat scenes ensuring that the volume does not affect the images acquisition. Imaging 3D objects would lead to phase differences (despite 


\section{take into consideration this aspect.}

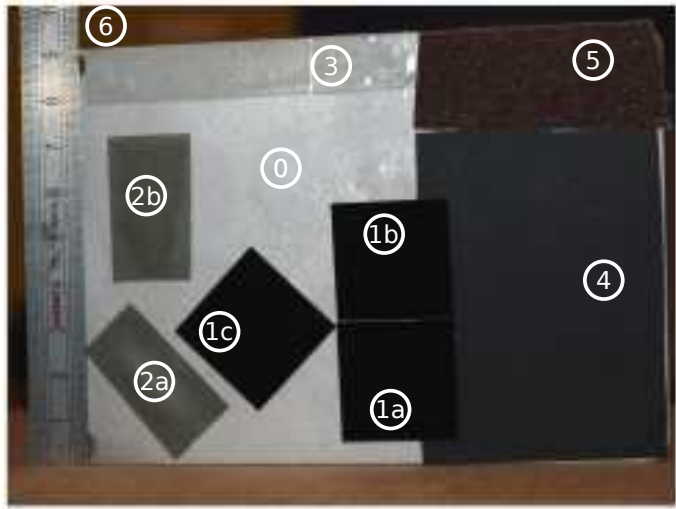

(a)

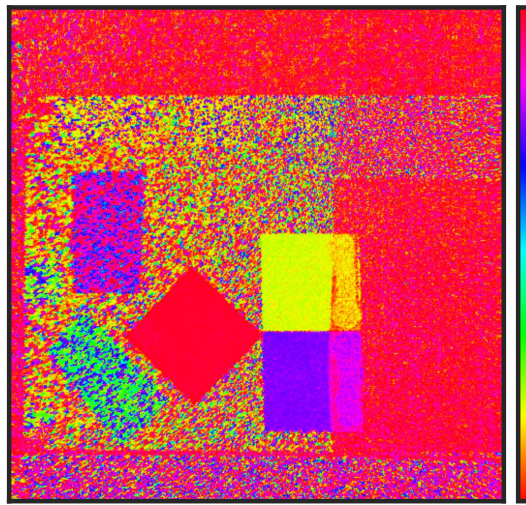

(c)

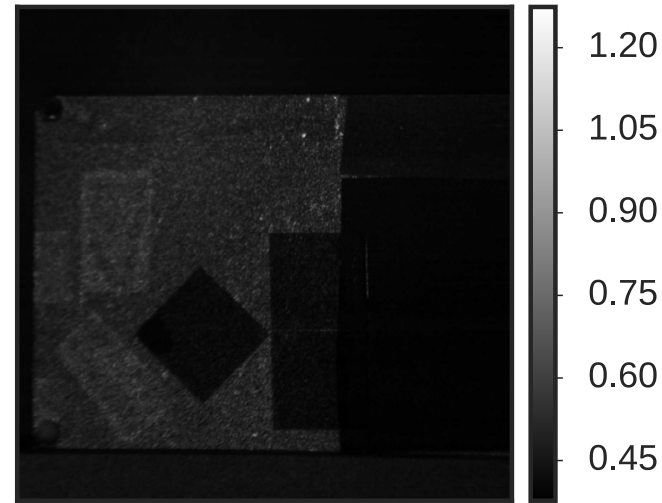

(b)

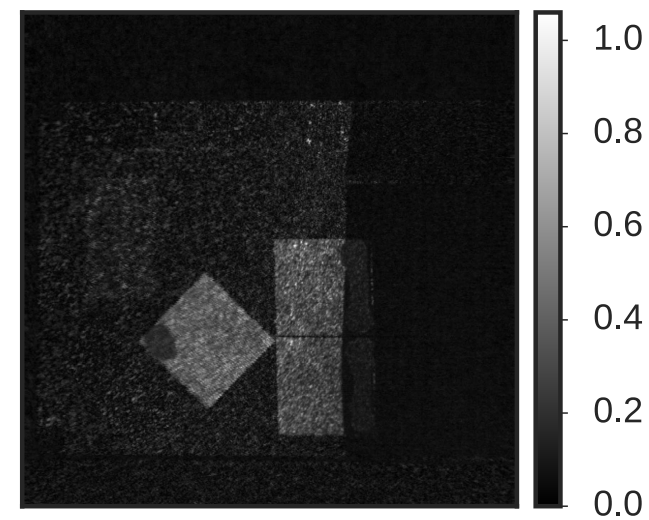

(d)

Fig 5 (a) Photograph of the test scene (captured with a visible CCD camera) comprising three IR polarizers (1), two visible polarizers (2), a double sided adhesive tape (3), a depolarizing black paper (4) and a sandpaper (5) on the right. The background is made of a metallic plate (0) and a wooden plank (6). DC (b), $\phi$ (c) and OBC (d) images obtained with the demonstrator and expressed respectively in volt, in degrees and in arbitrary unit.

DC, $\phi$ and OBC images are displayed respectively in Fig. 5(b-d). In the DC image, the metallic plate (0) appears lighter than the depolarizing elements (4 and 5) and the wooden plank (6) in the back, as the quantity of light sent back to the detector is higher. The birefringence of the adhesive tape (3) does not show contrasts with respect to the plate neither in the DC nor the OBC images. Since the orthogonality breaking technique is insensitive to birefringence effects, the absence of contrasts on the adhesive tape was expected. The two visible Polaroids ( $2 \mathrm{a}$ and $2 \mathrm{~b}$ ) are 
characterized simultaneously by a birefringent and a dichroic behaviors when used in the infrared range. As a result, slight contrasts can be observed in the phase map, as a weak modulation of the beatnote signal still implies a determined phase, measured locally. The three NIR Polaroids (1a, $1 \mathrm{~b}$ and 1c) are dark in the DC image (values close to $0.42 \mathrm{~V}$ ) but they are all revealed on the OBC map as they lead to strong orthogonality breaking signals. Their orientation is given by the phase map (see Fig. 5(c)) independently from their diattenuation coefficient that can be straightforwardly derived from OBC measurements. ${ }^{15,17}$ The OBC map is calibrated on a perfect polarizer while the detection stage of the imaging setup operates in its linear range. The Polaroids (1) are used to calibrate the imaging setup. According to their DC and $|\mathrm{AC}|$ values measured respectively to be $0.42 \mathrm{~V}$ and $0.15 \mathrm{~V}$, the detection stage operates linearly. The diattenuation coefficients of the Polaroids (1) are determined experimentally by measuring their minimum $\left(T_{m i n}\right)$ and maximum $\left(T_{\max }\right)$ transmittances $^{17}$ at $\lambda=1.55 \mu \mathrm{m}: 0.984(1 \mathrm{a}), 0.995(1 \mathrm{~b})$ and 0.984 (1c). Regarding the anisotropy orientation of the samples, they can be retrieved from their phase value through the following equation: ${ }^{15}$

$$
\psi_{1 i}=\left(\pi / 2-\phi_{1 i}\right) / 2
$$

with $\psi_{1 i}$ and $\phi_{1 i}$, respectively, the anisotropy orientation of the sample (1i) and its corresponding measured phase. The phase measured on the Polaroids (1a, $1 \mathrm{~b}$ and $1 \mathrm{c}$ ) are respectively: $\phi_{1 a}=96^{\circ}$, $\phi_{1 b}=-110^{\circ}, \phi_{1 c}=172^{\circ}$ corresponding to their respective orientations: $\psi_{1 a}=-3^{\circ}, \psi_{1 b}=100^{\circ}$ and $\psi_{1 c}=-41^{\circ}$. These results validate the theoretical adequacy of such a system to reveal dichroic effects from a single laser scan of the scene. 


\subsection{Spatial resolution}

Another important specification of an imaging setup is its instantaneous field-of-view (iFOV), or resolving power, which defines the angle covering the part of the scene integrated by a single pixel of the images. The spatial resolution, i.e. the size of the the scene corresponding to one pixel of the image, can then be deduced from the $\mathrm{FFOV}$ and the distance between the imaging system and the scene.

The iFOV is estimated at a distance of $2 \mathrm{~m}$ from the beam-forming optics by laser scanning an USAF resolution test target (R3L3S1N, Thorlabs). A NIR polarizer has been inserted in front of the detector to generate a beatnote signal that allows simultaneous measurement of the iFOV in DC and $|\mathrm{AC}|$ images. Six sets of acquisitions have been performed with an increasing number of pixels in the images while preserving the same FOV $\left(45 \operatorname{mrad} \times 45 \operatorname{mrad}\left(2.6^{\circ} \times 2.6^{\circ}\right)\right)$. From these images, the minimum resolvable group of the USAF target is determined and afterwards the IFOV is calculated taking into account the $2 \mathrm{~m}$ distance. Figure 6(a) plots the evolution of the iFOV versus the number of pixels in the images. As an example, $\mathrm{DC}$ and $|\mathrm{AC}|$ images acquired on the resolution test target, with 800 x 800 pixels, are displayed in Fig. 6(b). Beyond 512 x 512 pixels, the iFOV remains globally below $0.35 \mathrm{mrad}\left(0.02^{\circ}\right)$ in both images studied with the scene, placed at a distance of $2 \mathrm{~m}$. The spatial resolution in both directions of the transverse plane is thus $0.7 \mathrm{~mm}$ at a distance of $2 \mathrm{~m}$.

In Sec. 3.3, the noise affecting the temporal raw signals was studied. When the light beam is displaced over the scene, the signals acquired may be dominated by a noise different from the previous analysis. Thus, the next section is focused on the nature of the spatial noise that affects the images. 


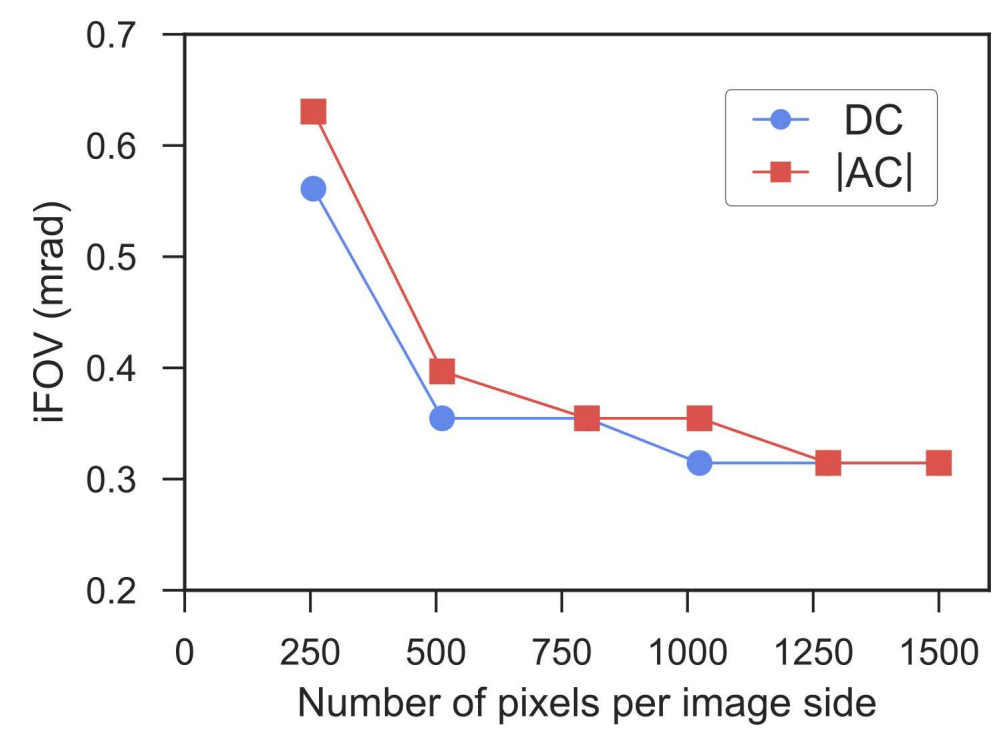

(a)

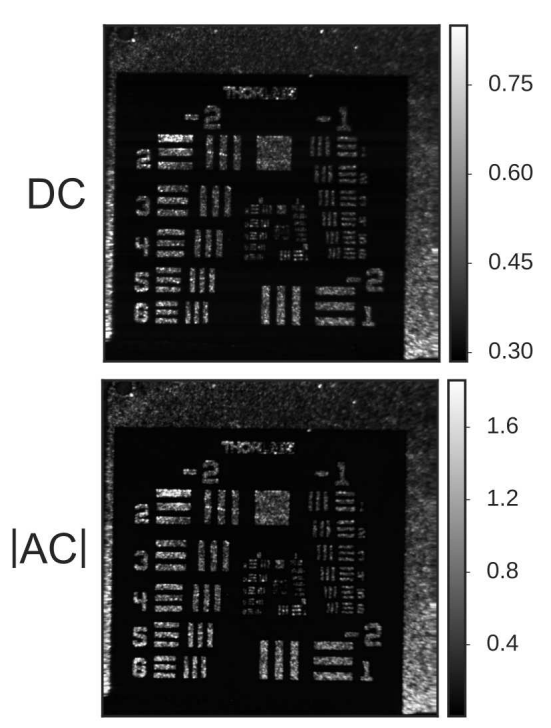

(b)

Fig 6 (a) Evolution of the demonstrator iFOV versus the number of pixels in the DC and $|\mathrm{AC}|$ images while keeping a constant field-of-view of $45 \mathrm{mrad}$ x $45 \mathrm{mrad}$. (b) Acquisition of DC and $|\mathrm{AC}|$ images (800 x 800 pixels).

\subsection{Analysis of the noise in the images}

To analyze the nature of the noise in the images acquired by the setup of Fig. 1, the images were segmented into homogeneous regions in order to estimate the probability density function (pdf) from a sufficient amount of pixels (several thousands typically). This segmentation into homogeneous regions was performed automatically using the unsupervised minimal description length active grid segmentation technique proposed in Ref. 20,21 and which relies on a non parametric modeling of the pdf of the grey levels inside each region. ${ }^{22,23}$ The partition into homogeneous regions obtained on the images of Fig. 5 are presented in Fig. 7 for images I, Q, |AC|, OBC. Similar results are obtained for the phase image (Figure 5(c)), but in that case, the result for the DC image (not displayed) was over-segmented.

The metallic plate (0), the IR polarizers (1), the black paper (4), the sand paper (5) and the wooden plank (6) are well identified as homogeneous regions in all the images. The visible polarizers (2) are both visible in the phase image (Figure 5c), whereas the polarizer (2a) is only detected 


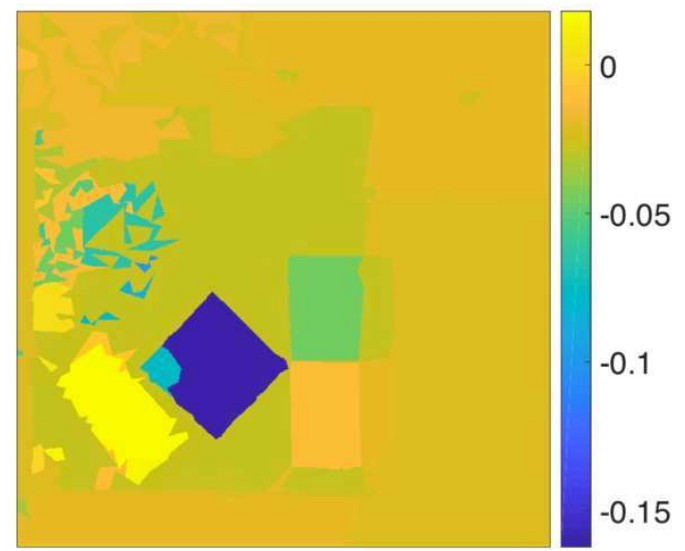

(a)

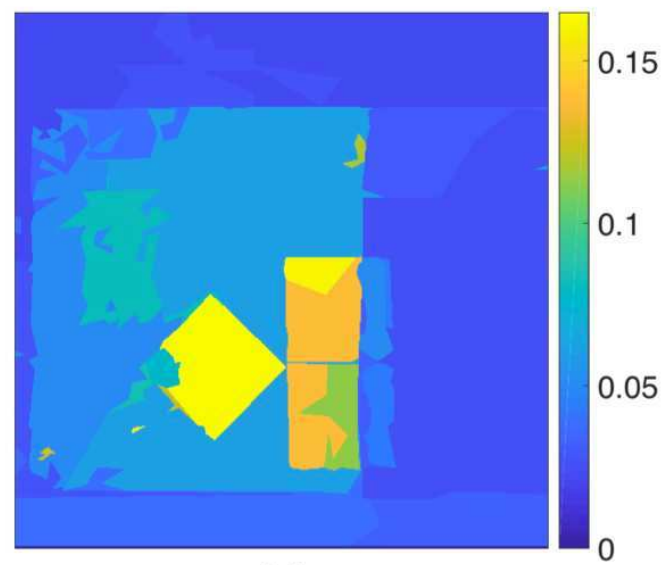

(c)

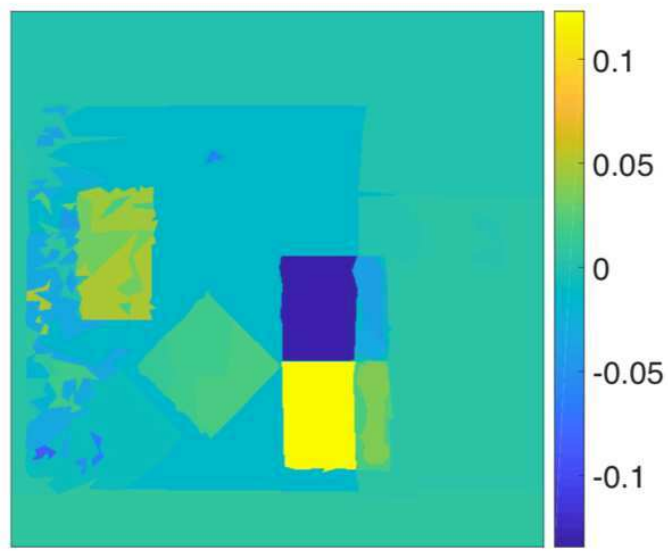

(b)

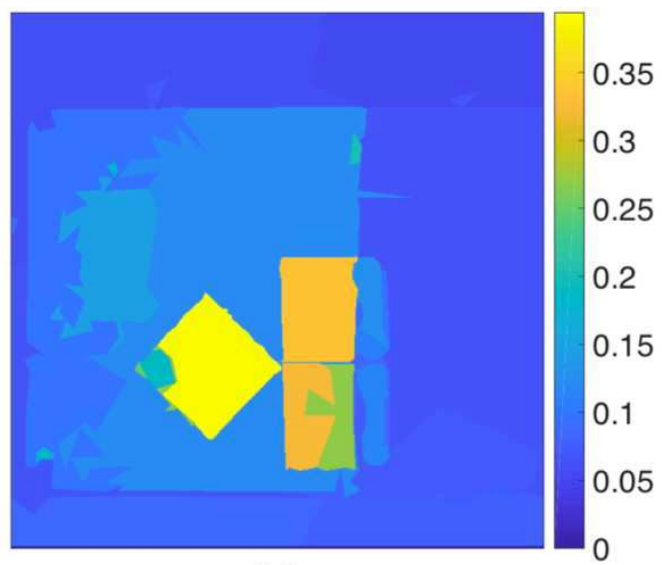

(d)

Fig 7 Results of the segmentation into homogeneous regions of the images of Fig. 5 using the minimum description length segmentation technique based on a non parametric modelling of grey level fluctuations. (a) Image I. (b) Image Q. (c) Image $|\mathrm{AC}|$. (d) Image $\mathrm{OBC}$. Each color corresponds to the mean value of the image in the considered homogeneous region.

as an homogeneous region in the segmented image of I. For the polarizer (2b), it appears in all the images except I. The homogeneous regions $R_{j}$ contain at least 3000 pixels except for the DC image. In the following, we focus on the region containing the infrared polarizer (1c) to carry out a statistical analysis of the noise properties. The given conclusions can be extended to the other objects.

The noise affecting the images I, Q and DC can be approximated for almost the considered 
region $j$ by a Gamma law of pdf: ${ }^{24}$

$$
p_{R_{j}} j\left(x_{i}\right)=\frac{x_{i}^{\alpha_{j}-1}}{\beta_{j}^{\alpha_{j}} \Gamma\left(\alpha_{j}\right)} e^{-x_{i} / \beta_{j}},
$$

with $x_{i}=\operatorname{pixel} i$ in the considered region $R_{j}, \alpha_{j}$ and $\beta_{j}$ the parameters of the Gamma law for the same region. This approximation is in agreement with the scattering nature of the sample, which, when illuminated by laser light, causes a speckle noise typical of active coherent imaging systems. $^{25}$

Histograms of the segmented region corresponding to the infrared polarizer (1c) for the images I, Q, DC are represented in Fig. 8 and are fitted with a Gamma distribution (black line with stars). The I and Q images have been processed to be positive (translating all values by adding the minimum value) so that the data could be fitted with Gamma laws. Indeed, the expression of a Gamma law is positively defined. ${ }^{24}$ The parameters of the law $\left(\alpha_{j}, \beta_{j}\right)$ are estimated by maximizing the likelihood of a Gamma process and are given in the legend of Fig. 8.
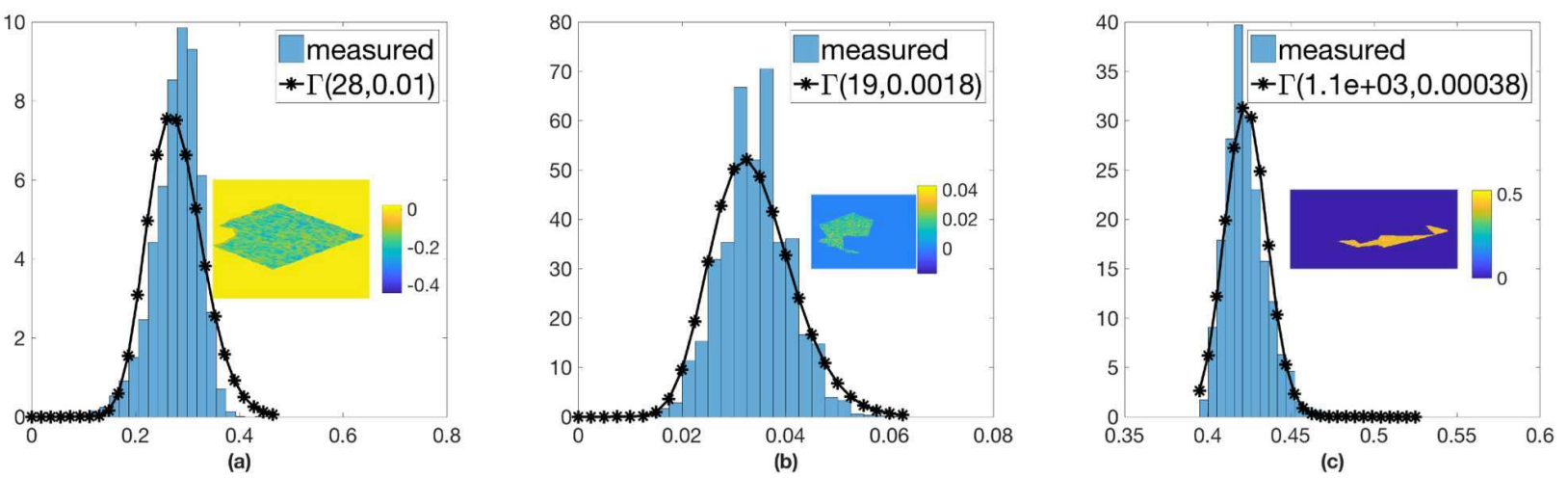

Fig 8 Histograms (25 bins) of the segmented region corresponding to the visible polarizer (1c). (a) Image I. (b) Image Q. (c) Image DC. The insets correspond to the considered segmented region for the histogram. Each histogram is fitted (black line with stars) by a Gamma law of parameters $\left(\alpha_{j}, \beta_{j}\right)$ specified in the legend.

Assuming Gamma laws for I, Q and DC does not give simple expressions for the statistical laws followed by $|\mathrm{AC}|$ and $\mathrm{OBC}$ signals. However, fitting the histogram of the $|\mathrm{AC}|$ regions of the 


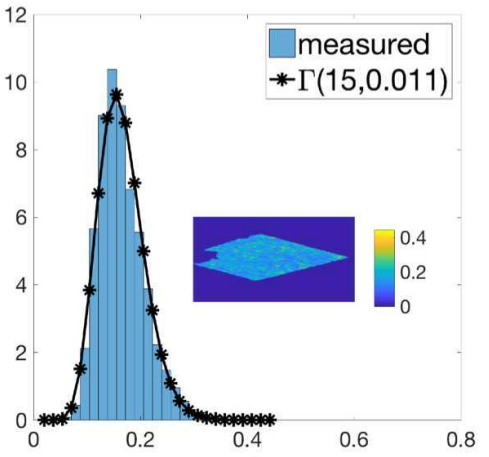

(a)

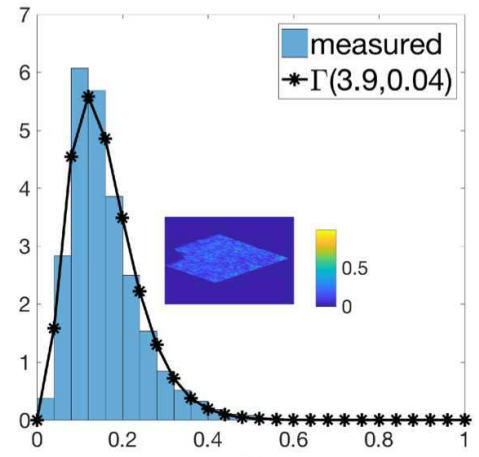

(b)

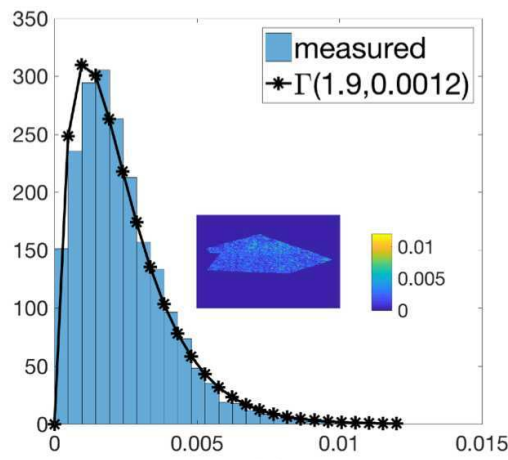

(c)

Fig 9 Histograms (25 bins) of the segmented region corresponding to the visible polarizer (1c). (a) Image $|\mathrm{AC}|$. (b) Image $\mathrm{I}^{2} / \mathrm{DC}^{2}$. (c) Image $\mathrm{Q}^{2} / \mathrm{DC}^{2}$. The insets correspond to the considered segmented region for the histogram. Each histogram is fitted (black line with stars) by a Gamma law of parameters $\left(\alpha_{j}, \beta_{j}\right)$ specified in the legend.

object shows that $|\mathrm{AC}|$ can be well approximated by a Gamma law (see Fig. 9(a)).

The OBC image can be rewritten as:

$$
\mathrm{OBC}=\sqrt{\frac{\mathrm{I}^{2}}{\mathrm{DC}^{2}}+\frac{\mathrm{Q}^{2}}{\mathrm{DC}^{2}}}
$$

Analyzing the histograms of images $\mathrm{I}^{2} / \mathrm{DC}^{2}$ and $\mathrm{Q}^{2} / \mathrm{DC}^{2}$ (see Fig. 9(b) and (c)), we found that we could fairly assume that they follow also Gamma laws of parameters $\left(\alpha_{k}, \beta_{k}\right)_{R_{j}}$ with $k=1$ for $\mathrm{I}^{2} / \mathrm{DC}^{2}$, and $k=2$ for $\mathrm{Q}^{2} / \mathrm{DC}^{2}$ for all the regions $R_{j}$ considered. This assumption allows $\mathrm{OBC}^{2}$ to be defined as the sum of two random variables following Gamma laws of different parameters. Assuming that these variables are independent, an exact expression of the pdf followed by $\mathrm{OBC}^{2}$ can be obtained, ${ }^{26}$ but this expression implies the computation of an infinite sum which is complex to manipulate. The sum of two Gamma laws can be approximated by a Gamma law ${ }^{27}$ of parameters $\alpha_{R_{j}}=\mu_{R_{j}}^{2} / \sigma_{R_{j}}^{2}$ and $\beta_{R_{j}}=\sigma_{R_{j}}^{2} / \mu_{R_{j}}$ where $\mu_{R_{j}}=\sum_{k=1}^{2}\left(\alpha_{k}\right)_{R_{j}} \times\left(\beta_{k}\right)_{R_{j}}$ and $\sigma_{R_{j}}^{2}=\sum_{k=1}^{2}\left(\alpha_{k}\right)_{R_{j}} \times$ $\left(\beta_{k}\right)_{R_{j}}^{2}$. This approximation is valid when $\left(\alpha_{k}\right)_{R_{j}}>0.1$ and $\left(\beta_{k}\right)_{R_{j}}$ differs by no more than a factor 10 (which is the case in our data). Finally, an approximation of the law followed by the OBC is given by a Nakagami law, ${ }^{28}$ which can be used to model the statistical distributions of the square 
root of a Gamma-distributed random variable. This is shown in Fig. 10(a-b).

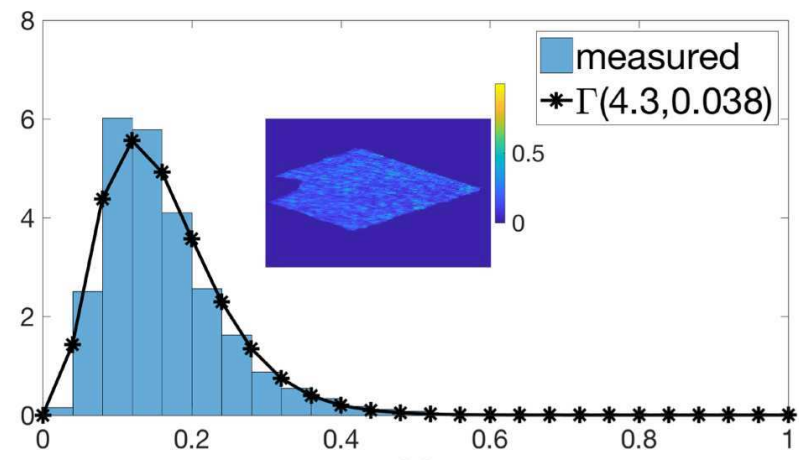

(a)

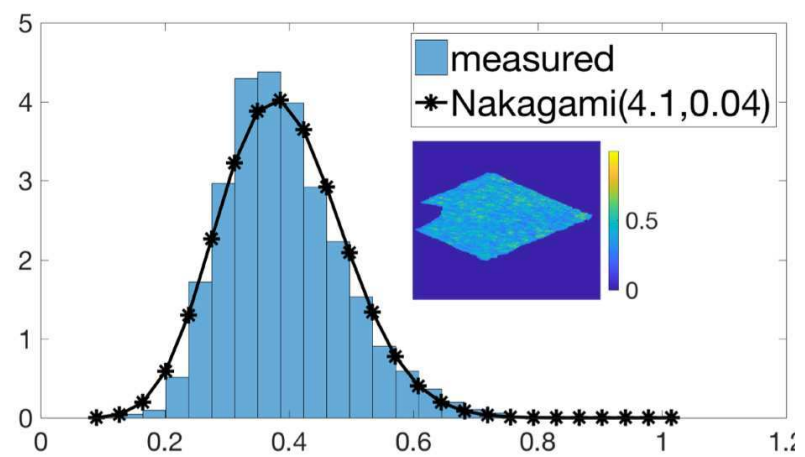

(b)

Fig 10 Histograms (25 bins) of the segmented region corresponding to the visible polarizer (1c). (a) Image OBC ${ }^{2}$. (b) Image OBC. The insets correspond to the considered segmented region for the histogram. Histogram (a) (respectively (b)) is fitted (black lines with stars) by a Gamma law of parameters $\left(\alpha_{k}, \beta_{k}\right)$ specified in the legend (respectively by a Nakagami law with parameters $\left(\alpha_{R_{j}}, \beta_{R_{j}}\right)$ ).

In the majority of cases encountered in the active imaging field using a highly coherent light source, the quality of the images is degraded by speckle noise. ${ }^{25}$ In the following, we analyze the influence of the temporal coherence of the DFDP source on the noise that dominates the images acquired with the imaging setup.

\section{Influence of the source coherence}

The range of such an imaging system could be improved by taking advantage of pulsed sources which feature lower temporal coherence (larger spectral width reciprocally) than the front Tunics laser's. In order to analyze the influence of the DFDP source coherence on the images, the validity of the results is first evaluated when the wavelength of the Tunics laser is tuned around $1550 \mathrm{~nm}$. Then, experimental results will be compared between three DFDP sources with different temporal coherence. 


\subsection{Demonstrator operation with a tunable source}

So far, the imaging setup has been investigated with a highly coherent source emitting a DFDP light beam at $1550 \mathrm{~nm}$. The tunability of the front laser over the C-L telecom bands (1500 to $1630 \mathrm{~nm}$ ) is used to determine the wavelength range over which the imaging system can be used without degrading the performances. This range will dictate the maximum spectral width that can be considered for the low coherent DFDP sources tested in Sec. 5.2.

For this purpose, the front laser wavelength is tuned over its available range: $1500-1630 \mathrm{~nm}$ ( $\mathrm{C}$ and $\mathrm{L}$ bands). In this interval, important changes in the source optical power and polarization states appear. On one hand, the spontaneous emission of the EDFA shows a strong dependence on the wavelength, as depicted in Fig. 11. The filled area indicates the wavelength range (1530$1575 \mathrm{~nm}$ ) over which the gain of the EDFA is sufficient to provide a maximum output optical power of $20.5 \mathrm{dBm}$. To highlight the impact of the EDFA gain variations on the measurements, the RF power delivered by the fibered photodiode from the reference block has been measured at multiple discrete wavelengths lying between 1500 and $1630 \mathrm{~nm}$. As illustrated in Fig. 11, over the spectral range $1530-1575 \mathrm{~nm}$, the RF power is maintained at $-26.5 \pm 0.5 \mathrm{dBm}$ despite residual variations caused by residual optical power and polarization state changes. As a consequence, the acquisitions should be limited to the range 1530-1575 $\mathrm{nm}$ to warrant a constant DFDP source optical power.

On the other hand, it was also observed that the DFDP source polarization states (which should be linearly polarized as was the case at $\lambda=1.55 \mu \mathrm{m}$ ) were disturbed when the wavelength was varied. This effect was analyzed with a commercial Stokes polarimeter (PAT9000B, Thorlabs), which showed that the 90:10 fibered splitter was mainly responsible for the gain of ellipticity 


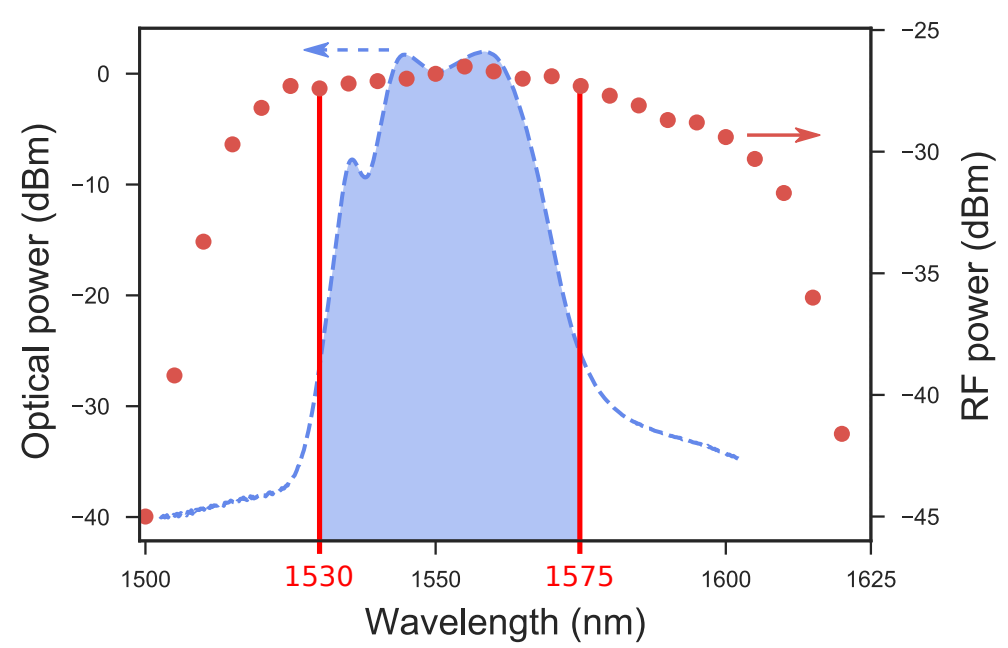

Fig 11 Experimental spontaneous emission of the EDFA (blue dashed curve) measured with an optical spectrum analyzer (resolution: $0.2 \mathrm{~nm}$ ). The filled blue area shows the range in which an input signal is amplified to a constant optical power of $20.5 \mathrm{dBm}$. Experimental magnitude of the beatnote signal at $80 \mathrm{MHz}$ (red circle markers) delivered by the fibered photodiode from the reference block for different wavelengths of the front laser.

observed on the emitted state of polarization when the wavelength was varied.

To reduce this defect in a tunable wavelength operation mode, the 90:10 splitter can be removed and the fibered reference block used to generate the LO signal in the demodulation process can be replaced by an equivalent free space arrangement. This configuration was tested by focusing the reflection of the light source on the 50:50 BS (placed after the collimator) on a high-speed photodiode (EPITAXX ETX 300) through a NIR AR coated lens. A beatnote signal was produced by inserting a polarizer in the path of light before its detection. The quantity of light detected was controlled by a circular aperture, so that the $80 \mathrm{MHz}$ RF power of the LO signal could be set to its optimal value of $-26 \mathrm{dBm}$. This small aperture iris also contributes to prevent reflections on the polarizer, the lens and the photodiode from being collected by the APD module.

These modifications of the imaging system enable valid measurements to be performed when the front laser wavelength is varied in the range 1530-1575 $\mathrm{nm}$. To confirm this statement, a test scene was imaged at several wavelengths. In this test scene, three NIR Polaroids are concealed among black patterns printed onto a white depolarizing sheet placed at a distance of $2 \mathrm{~m}$ from the 
imaging system. Five scans of the scene are performed with a circularly polarized DFDP source, its wavelength being consecutively varied from 1530 to $1570 \mathrm{~nm}$ with $10 \mathrm{~nm}$ steps. Figure 12 shows the images obtained at three different wavelengths: 1530, 1540 and $1560 \mathrm{~nm}$.

(a)
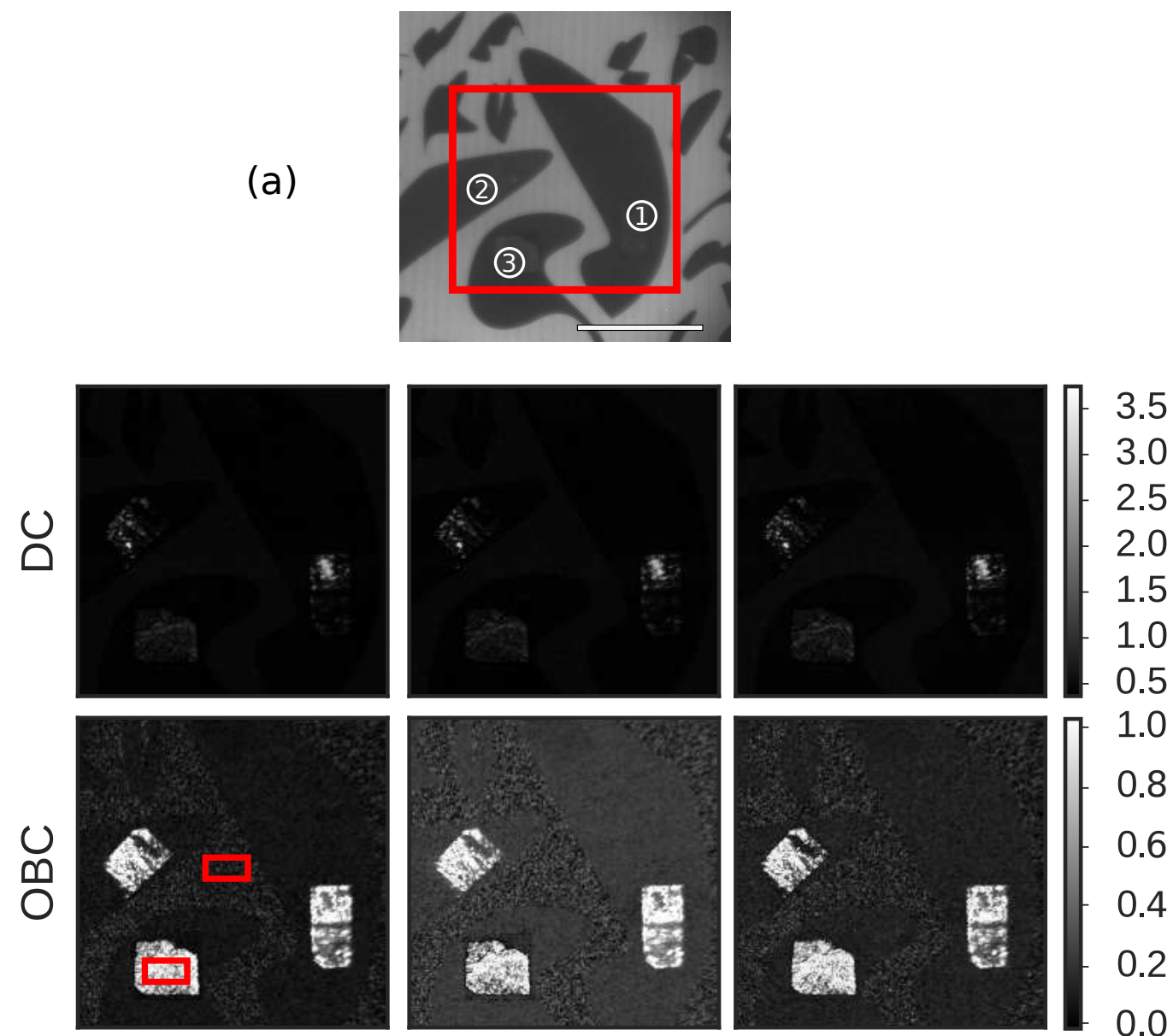

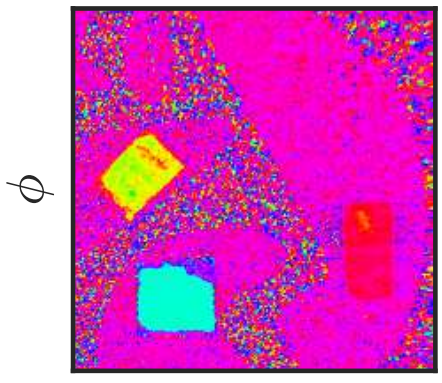

(b)

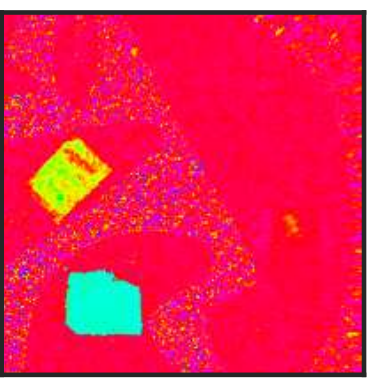

(c)

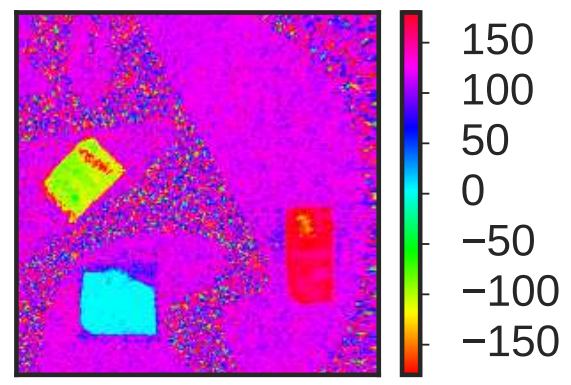

(d)

Fig 12 (a) Photograph of the scene (captured with an InGaAs CCD camera) where the red rectangle points out the scanned area and the white scale bar represents $50 \mathrm{~mm}$. Three NIR polarizers are hidden among black patterns printed on a white paper sheet. DC (top row), OBC (middle row) and phase $\phi$ (bottom row) images acquired at three different wavelengths : $1530 \mathrm{~nm}$ (a), $1540 \mathrm{~nm}$ (b) and $1560 \mathrm{~nm}$ (c). Red rectangular homogeneous regions (70 x 35 pixels) are used to calculate the spatial OBC mean values which are displayed in Fig. 13. 
A quick overview of the DC and OBC images indicates that all three polarizers are similarly evidenced at the three wavelengths. Likewise, their phase values are preserved as the wavelength of the laser is shifted. However, the $\mathrm{OBC}$ and phase values undergo changes on the depolarizing background due to residual variations of the RF power of the LO signal.

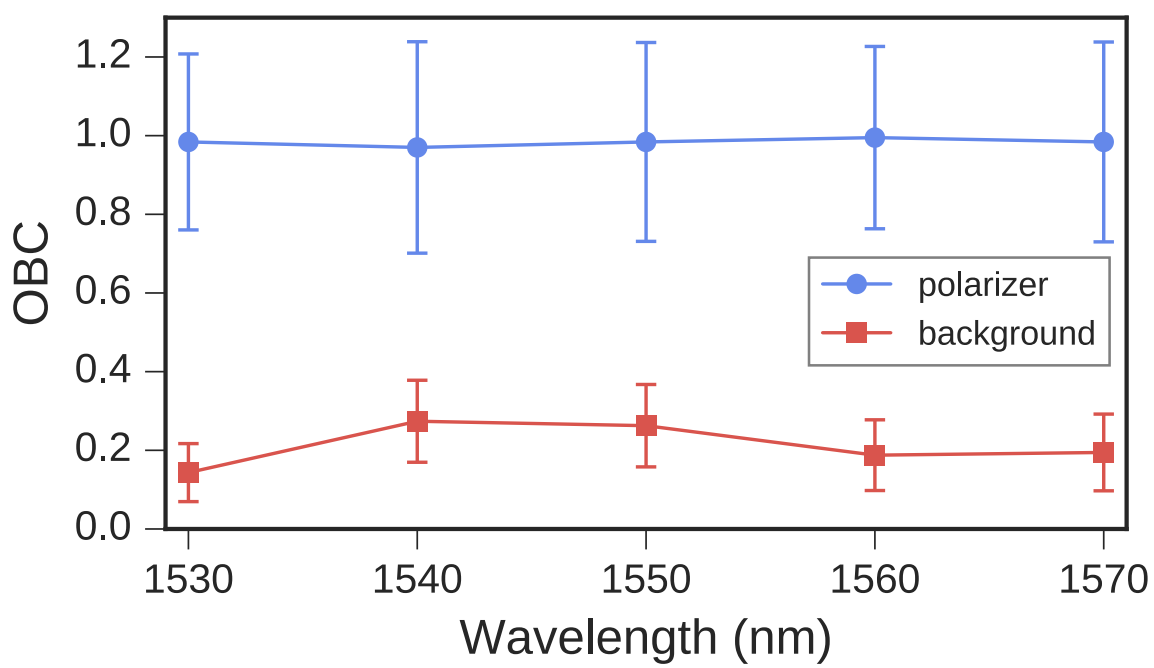

Fig 13 OBC mean values calculated on delimited areas of 70x35 pixels [red rectangles in OBC image from Fig. 12(b)] on a polarizer (3) and a part of the white background. The error bars are derived from the standard deviation of all pixels comprised in the regions of interest.

To illustrate this observation, the OBC mean values are plotted versus the wavelength on two rectangular areas, respectively on a polarizer (3) and a part of the white background. These regions are highlighted by red rectangles in the OBC image of Fig. 12(b). As shown in Fig. 13, the contrast varies slightly with the wavelength, most probably due to a bias in the measured beatnote amplitude $(|\mathrm{AC}|)$ caused by variations of the LO RF power. Nonetheless, the OBC and phase values remain constant, thus validating the capability of such a demonstrator to evidence contrasts on dichroic objects over the 1530-1570 nm range. It must be noted that changes of the $\mathrm{OBC}$ and phase values could have been observed when the wavelength is varied due to an evolution of the polarimetric response of the scene over the probed wavelength range. However, this is not the case here, and generally most scenarios may not display strong variations of their polarimetric response over such 
a narrow bandwidth.

The experimental results have so far shown that the acquisition of images performed in the 1530-1575 $\mathrm{nm}$ range yielded a fairly similar polarimetric contrast. Thus, results at any single wavelength within this range are equally valid, because the polarimetric responses of both the illumination/detection system and the scene are similar. If a broadband source is now used instead of a narrow source, the measurement becomes an integral of the outcome for each individual wavelength in the broad spectrum. As the beatnote signal differences are negligible within this range, the integrated images remain valid. As a consequence, sources with large spectral width, i.e. low temporal coherence, can be employed to acquire the polarimetric response of the scene.

\subsection{Influence of the temporal coherence of the source}

Section 4 has shown that the raw images were dominated by speckle noise. As this noise could originate from the high coherence of the DFDP source, the influence of the source temporal coherence on the noise affecting the raw images is studied. Furthermore, imaging results will be compared with three front sources exhibiting different temporal coherence values that are summarized in Tab. 1.

As discussed in Sec. 2.1, the temporal coherence of the front light source may affect the demodulation process if the arms of the Mach-Zehnder architecture, producing the DFDP beam, have unbalanced lengths. In this regard, the tunable optical delay line (ODL) (inserted inside the DFDP module) allows the temporal delay between the arms length to be balanced with a maximum precision of $1 \mathrm{fs}$ (i.e., a fiber length of $0.2 \mu \mathrm{m}$ ). According to the sources temporal coherence estimated from experimental measurement and provided in Tab. 1, it can be noticed that the coherence of the source 1 is the smallest with a value of 0.4 ps. Using this source, the DFDP module arms length 
Table 1 Spectral features of the three sources: FWHM (full width at half maximum), temporal coherence $\tau_{C}$ and its corresponding coherence length $L_{C}$. The central wavelength is $1550 \mathrm{~nm}$.

\begin{tabular}{|c|l|c|c|c|}
\hline Number & Source description & FWHM $(\mathrm{nm})$ & $\tau_{C}(\mathrm{ps})$ & $L_{C}(\mathrm{~mm})$ \\
\hline 1 & $\begin{array}{l}\text { Amplifier ASE (Keopsys, } \\
\text { KPS-BT-C21-Bo-FA) + EDFA }\end{array}$ & 20 & 0.4 & 0.083 \\
\hline 2 & Amplifier ASE + optical filter + EDFA & 0.7 & 11.3 & 2.34 \\
\hline 3 & Tunics laser + EDFA & $<3.10^{-6}$ & $>2.2 .10^{6}$ & $>5.52 .10^{5}$ \\
\hline
\end{tabular}

could be balanced accurately with a delay close to 10 fs (i.e. $2 \mu \mathrm{m}$ ) introduced by the ODL. Thus, the DFDP source remains well balanced with all three considered front sources.

The influence of the DFDP source temporal coherence on the noise affecting the raw images is now studied. Acquisitions are performed with the three sources while imaging a homogeneous metallic plate to enable the analysis of the dominating noise on a large amount of pixels. A polarizer has been introduced in front of the detector such that a beatnote signal is generated and the noise affecting the I and Q images can be evaluated simultaneously with the DC image. As in Sec. 4.5, histograms derived from homogeneous areas in the images are exploited to identify the nature of the dominant noise. Figure 14 displays the DC, I and Q histograms (50 bins) obtained with the three sources.

The fair agreement of all histograms with Gamma distributions (in dotted lines) reveals that a speckle noise remains dominant even though the source temporal coherence is decreased. This result was expected as the speckle noise is not only induced by the temporal coherence of the DFDP source but also by its spatial coherence ${ }^{29}$ in the confocal configuration.

Experimentally, the consistency of the results interestingly indicates that the imaging system may operate quite similarly with a high or a low coherent front light source. This is illustrated in Fig. 15 using the scene of Fig. 12. As the DFDP source is changed, no apparent modification 

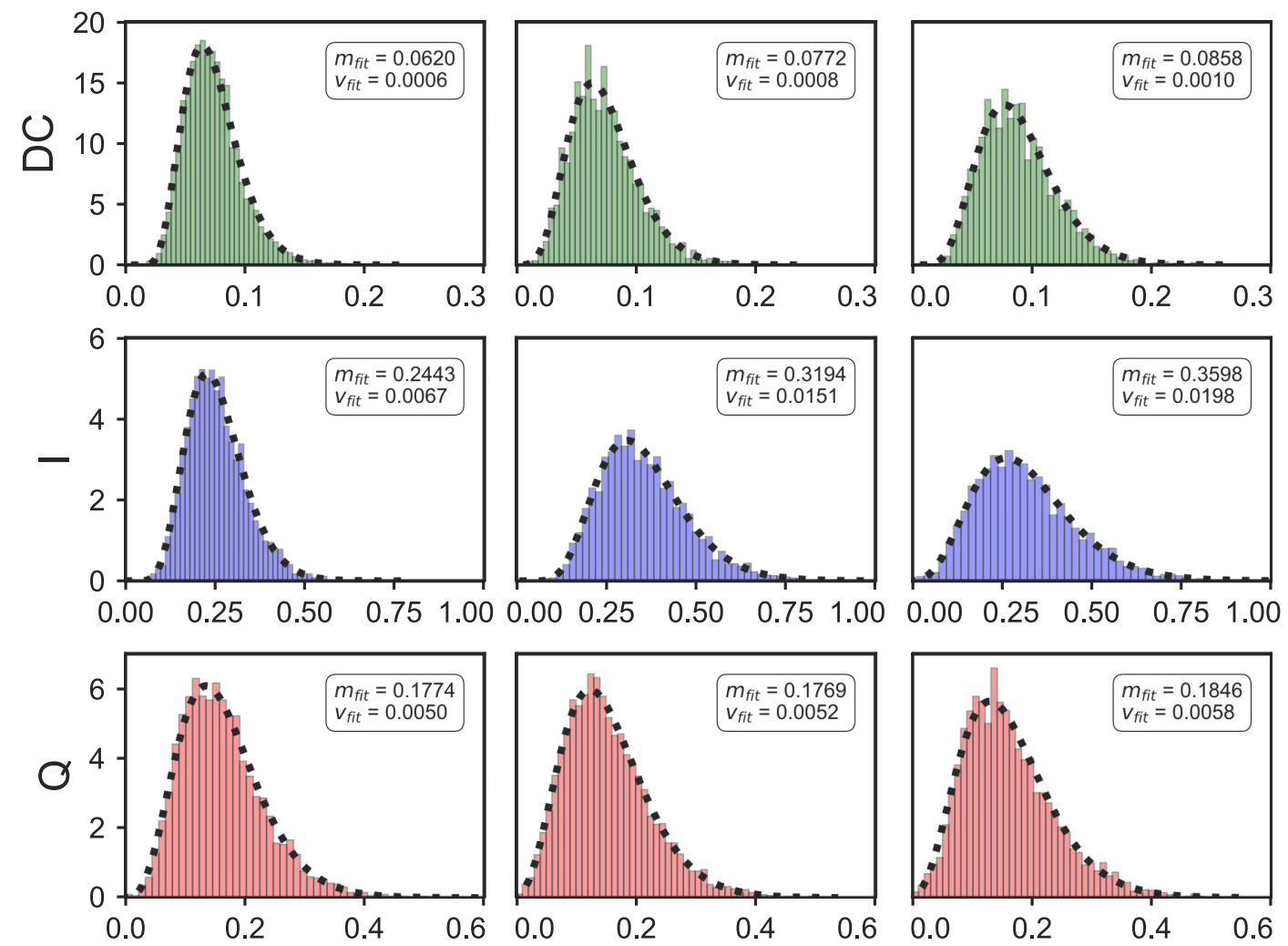

(a)

(b)

(c)

Fig 14 DC (top row), I (middle row) and Q (bottom row) (in volt) histograms (50 bins) calculated from $105 \times 45$ pixels in the corresponding images on an homogeneous metallic plate enlightened by low coherent DFDP sources 1 (a) and 2 (b), and a highly coherent DFDP source 3 (c). The sources spectral features are summarized in Tab. 1. Each histogram is fitted with a Gamma distribution, superimposed in a black dotted line, which mean and variance values are given in insets.

can be observed in the DC and OBC images neither on the polarizers, nor the background. While the phase seems to be constant on the polarizers, it varies drastically in the background with the considered source. This is due to residual variations of the I and Q signals which are induced by low changes of the LO signal (when the source are changed) used in the demodulation process.

To illustrate these statements, the mean values of $\mathrm{OBC}$ and $\phi$ from two regions of interest (a polarizer (3) and a part of the white background) are plotted in Fig. 16 for the three sources. The OBC mean values remain fairly steady in both regions with a high value (about 0.98 ) on the polarizer and a low value (about 0.16) on the background area. The phase remains constant on the polarizer region since the high beatnote produced sets a specific phase. However, on the 

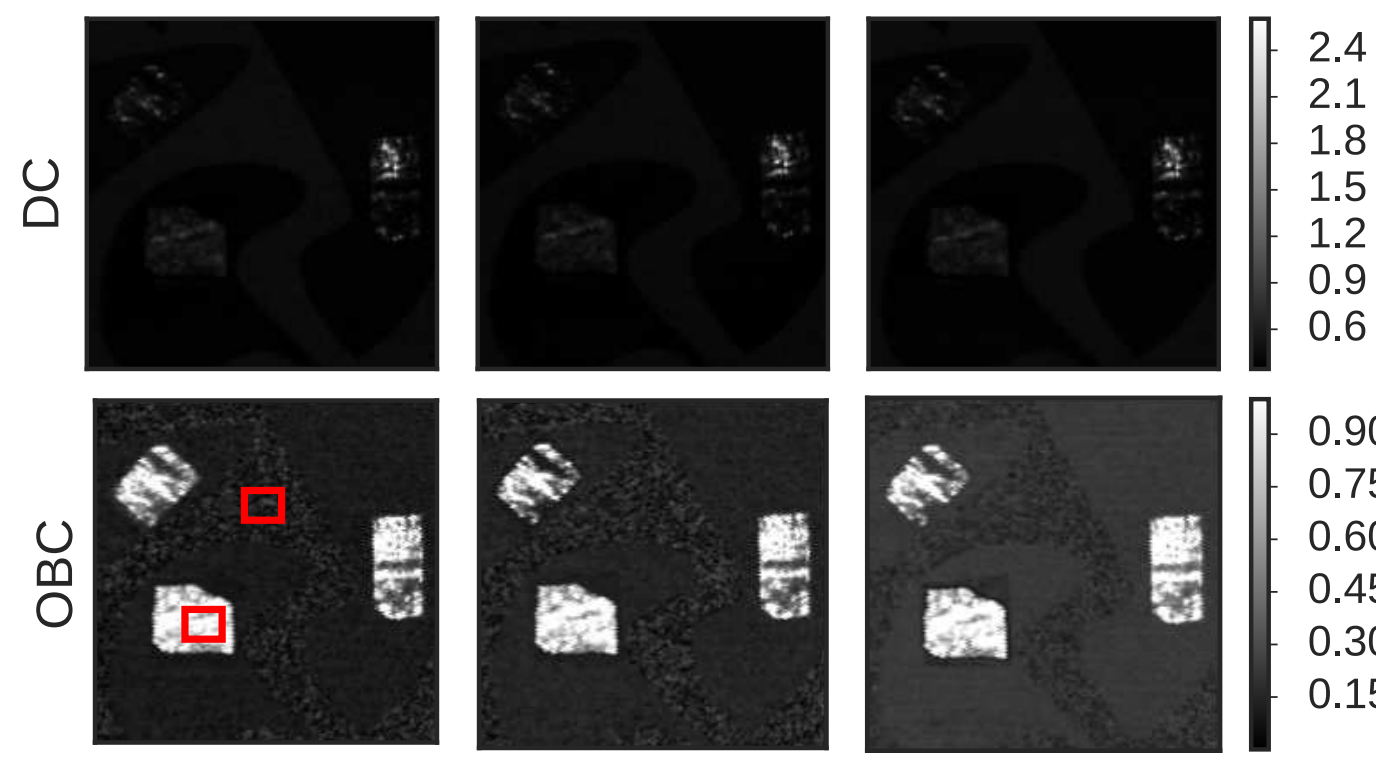

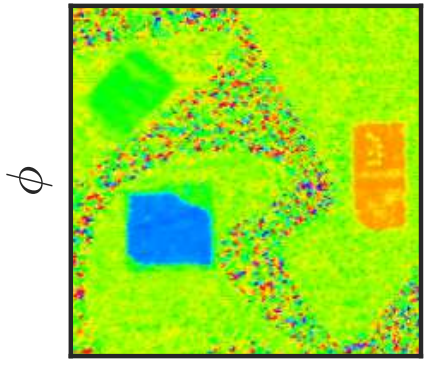

(a)

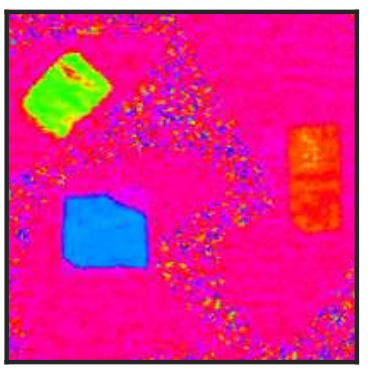

(b)

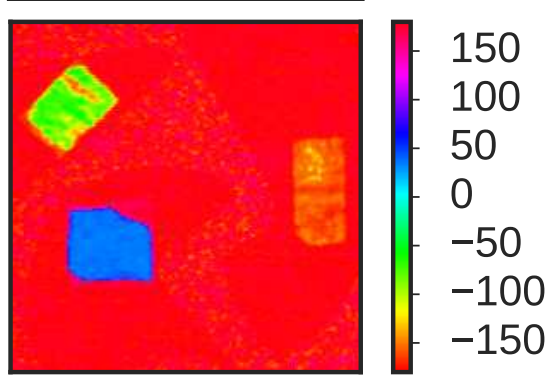

(c)

Fig 15 DC (top row), OBC (middle row) and $\phi$ (bottom row) images (230 x 230 pixels) of the test scene enlightened with the source 1 (a), the source 2 (b) and the source 3 (c). Red insets (20x25 pixels) indicate two regions (a polarizer and a part of the white background) used to analyze the influence of the coherence on the OBC and $\phi$ values, which are plotted in Fig. 16.

background area, the absence of beatnote leads to low I and Q signals which are subject to residual variations when the front source is changed. As a result, the phase mean value on the background is strongly disturbed showing a standard deviation spreading over $\pm 100^{\circ}$. The measurements remains nevertheless valid with the three sources despite phase variations (which could be calibrated for each source) observed on the background area. As a consequence, orthogonality breaking contrast images can still be acquired by the imaging system when the temporal coherence of the DFDP source is considerably reduced.

This result is interesting towards the development of an orthogonality breaking imaging system 


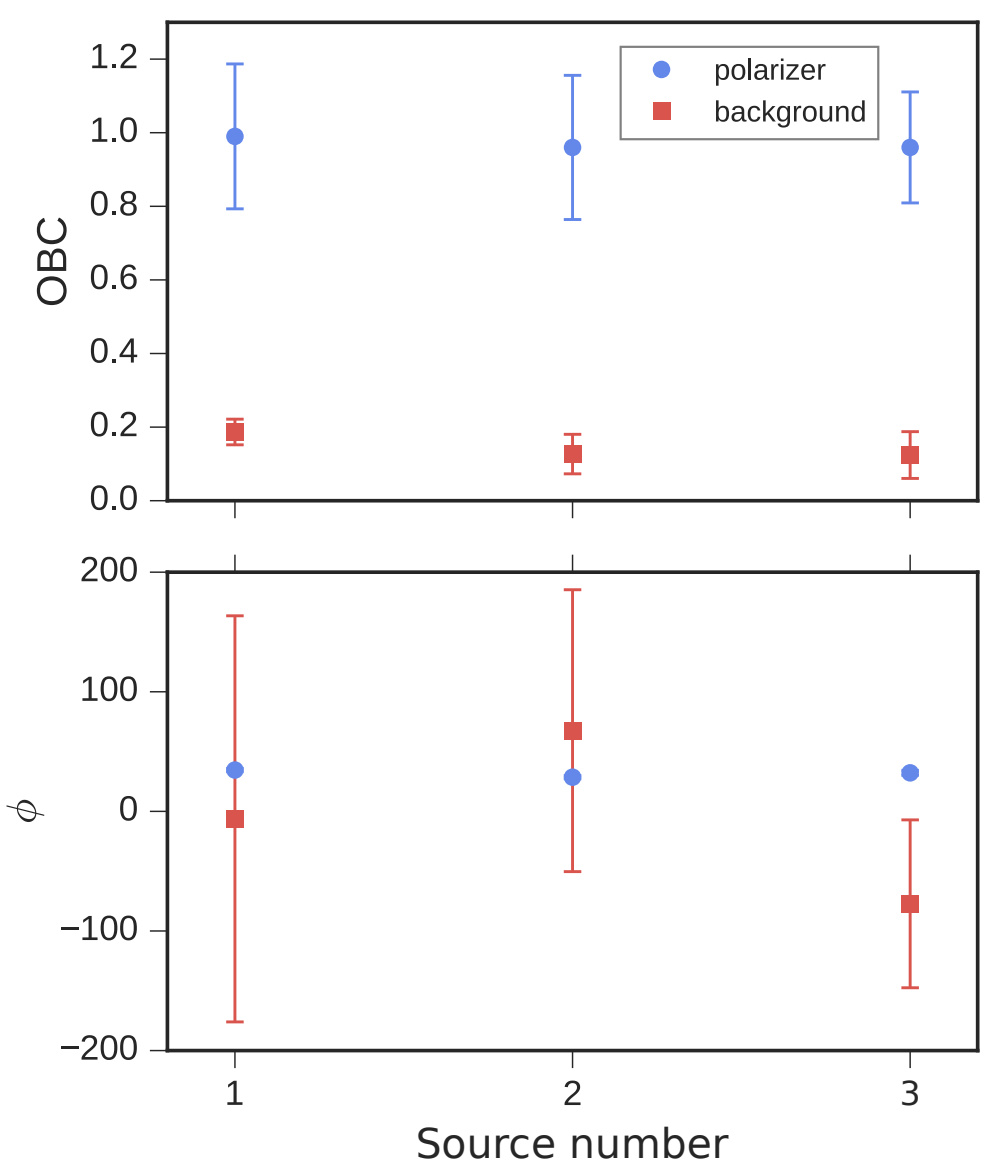

Fig 16 Evolution of the mean values of $\mathrm{OBC}$ and $\phi$ on a NIR polaroid (blue circle symbols) and a white area from the background (red square symbols) for the three sources. The error bars correspond to the standard deviation calculated from the 20x25 pixels in the regions of interest. Both regions of interest are indicated by red insets in Fig. 15.

based on a pulsed DFDP source ${ }^{30-32}$ for outdoor scenarios (range of hundreds of meters). Indeed, the extrapolation of the power budget indicates that imaging at $100 \mathrm{~m}$ would require, at least, a deposited power of $17.5 \mathrm{~W}$, thus implying a continuous front source of $285 \mathrm{~W}$ (if losses were to remain similar as the losses obtained with the DSOB imaging system described here) in the experimental configuration described in this article. A pulsed DFDP source, characterized by low temporal coherence, would enable imaging at long range to the expense of an increased complexity of the system (synchronization of the illumination and the detection). 


\section{Conclusion and outlooks}

In conclusion, we have detailed the design of an infrared active polarimetric imaging system based on the orthogonality breaking technique. Operating in a confocal configuration, the imaging setup exploits a $\sim 10 \mathrm{~mW}$ dual-frequency dual-polarization source at $\lambda=1.55 \mu \mathrm{m}$ used to scan a remote scene (up to several meters) in approximately $1 \mathrm{~s}$. The backscattered light is collected on an avalanche photodiode and the polarimetric images are built digitally from three raw signals: DC, I and Q. We have investigated the response of the detection stage and showed that it is linear for a detected power lying between 0.01 and $1 \mu \mathrm{W}$. We have also highlighted that the temporal raw signals are dominated by Gaussian noise. The complete imaging system has been then validated and calibrated on a test scenario with known polarimetric properties thus confirming its ability to reveal dichroic objects. The spatial noise in the raw images was analyzed, showing that fluctuations can be well modeled with Gamma distributions of low order. Such noise statistics are characteristic of (weakly-averaged) speckle noise, which could be expected with such a coherent active imaging system. Moreover, the pdf of the spatial fluctuations of the OBC image could be well modeled with a Nakagami law (which corresponds to the pdf of the square root of a Gamma-distributed random variable). We finally studied the influence of the source temporal coherence on the images. For this purpose, the wavelength of the DFDP source (highly coherent) has been first tuned so that the wavelength range that ensures the preservation of the system results could be determined experimentally to be between 1530 to $1575 \mathrm{~nm}$. This result showed that sources with large spectral width (low temporal coherence) up to $20 \mathrm{~nm}$ could be used without lowering the acquisition performance of polarimetric images. Then, the influence of the temporal coherence of the DFDP source on the noise has been compared for three different sources. This study revealed that the imaging system 
was operating very similarly, regardless of the temporal coherence of the DFDP source used.

As a first perspective to this work, the imaging system could be improved by delivering a reference signal with a constant RF power $(-26 \mathrm{dBm})$ at the LO demodulation input. This could be achieved, for instance, with a variable gain amplifier delivering a constant output power, or with a highly stable dual-frequency laser $^{33}$ instead of the fibered DFDP source we developed. Towards outdoor scenarios perspectives (range of hundreds of meters), a first approach would be based on the development of an imaging system using a pulsed DFDP light source at $\lambda=1.55 \mu \mathrm{m}$. An alternative approach would stand on improving the detection block, for instance by replacing the standard detection by an optical coherent detection system that would considerably increase the sensitivity of the imaging system. Lastly, this study paves the way for original polarimetric measurements by orthogonality breaking that would enable addressing and identifying other polarimetric effects than dichroism, such as birefringence or depolarization, which is presently under investigation.

\section{Acknowledgments}

Agence Nationale de la Recherche (ANR) (ANR- 13-ASTR-0001); Délégation Générale pour l'Armement. F.P.'s Ph.D. was supported by the DGA-MRIS scholarship and by Région Bretagne. The authors are grateful to Philippe Adam for fruitful discussions, Frédéric Galland and Philippe Réfrégier for their contribution to the noise analysis of the images. The authors are thankful to Steve Bouhier, Cyril Hamel and Emmanuel Schaub for technical support.

\section{References}

1 A. Pierangelo, S. Manhas, A. Benali, et al., "Multispectral Mueller polarimetric imaging detecting residual cancer and cancer regression after neoadjuvant treatment for colorectal 
carcinomas," Journal of Biomedical Optics 18(4), 046014 (2013).

2 T. Novikova, A. Pierangelo, S. Manhas, et al., "The origins of polarimetric image contrast between healthy and cancerous human colon tissue," Applied Physics Letters 102(24), 12-16 (2013).

3 F. Snik, J. Craven-Jones, M. Escuti, et al., "An overview of polarimetric sensing techniques and technology with applications to different research fields," in Proceedings of the SPIE, 9099, 90990B (2014).

4 L. Thomas, M. Boffety, and F. Goudail, "Improving target discrimination ability of active polarization imagers by spectral broadening," Optics Express 23(26), 33514-33528 (2015).

5 A. Le Gratiet, M. Dubreuil, S. Rivet, et al., "Scanning Mueller polarimetric microscopy," Optics Letters 41(18), 4336-4339 (2016).

6 P. Terrier, V. Devlaminck, and J. M. Charbois, "Segmentation of rough surfaces using a polarization imaging system.," Journal of the Optical Society of America A 25(2), 423-430 (2008).

7 S. L. Jacques, J. C. Ramella-Roman, and K. Lee, "Imaging skin pathology with polarized light.," Journal of Biomedical Optics 7(3), 329-340 (2002).

8 M. Alouini, F. Goudail, N. Roux, et al., "Active spectro-polarimetric imaging: signature modeling, imaging demonstrator and target detection," The European Physical Journal Applied Physics 42(2), 129-139 (2008).

9 S. Breugnot and P. Clemenceau, "Modeling and performances of a polarization active imager at $\lambda=806 \mathrm{~nm}$," Optical Engineering 39(10), 2681 (2000). 
10 N. Vannier, F. Goudail, C. Plassart, et al., "Comparison of different active polarimetric imaging modes for target detection in outdoor environment," Applied Optics 55(11), 2881-2891 (2016).

11 J. A. Zuclich, D. J. Lund, P. R. Edsall, et al., "High-power lasers in the 1.3-1.4 $\mu$ m wavelength range: ocular effects and safety standard implications," SPIE. Laser and noncoherent light ocular effects: Epidemiology, Prevention and Treatment 4246, 78-88 (2001).

12 R. L. McCally, C. B. Bargeron, J. A. Bonney-Ray, et al., "Laser eye safety research at APL," in Johns Hopkins APL Technical Digest, 26(1), 46-54 (2005).

13 F. Parnet, J. Fade, N. Ortega-Quijano, et al., "Free-space active polarimetric imager operating at 1,55 $\mu \mathrm{m}$ by orthogonality breaking sensing," Optics Letters 42(4), 723 (2017).

14 J. Fade and M. Alouini, "Depolarization remote sensing by orthogonality breaking," Physical

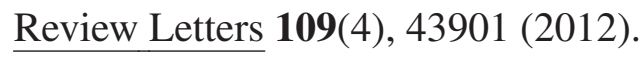

15 N. Ortega-Quijano, J. Fade, M. Roche, et al., "Orthogonality-breaking sensing model based on the instantaneous Stokes vector and the Mueller calculus," Journal of the Optical Society of America A 33(4), 434-446 (2016).

16 F. Parnet, J. Fade, and M. Alouini, "Orthogonality breaking through few-mode optical fiber," Applied Optics 55(10), 2508-2520 (2016).

17 N. Ortega-Quijano, J. Fade, E. Schaub, et al., "Full characterization of dichroic samples from a single measurement by circular polarization orthogonality breaking," Optics Letters 40(7), $1270-1273$ (2015).

18 N. Ortega-Quijano, J. Fade, F. Parnet, et al., "Generation of coherent light beam with precise 
and fast dynamic control of the state and degree of polarization," Optics Letters 42(15), 1-5 (2017).

19 F. Mufti and R. Mahony, "Statistical analysis of signal measurement in time-of-flight cameras,” ISPRS Journal of Photogrammetry and Remote Sensing 66(5), 720-731 (2011).

20 F. Galland, N. Bertaux, and P. Réfrégier, "Minimum description length synthetic aperture radar image segmentation,” IEEE Transactions on Image Processing 12(9), 995-1006 (2003).

21 F. Galland, A. Jaegler, M. Allain, et al., "Smooth contour coding with minimal description length active grid segmentation techniques," Pattern Recognition Letters 32(5), 721-730 (2011).

22 F. Galland and P. Réfrégier, "Minimal stochastic complexity snake-based technique adapted to an unknown noise model," Optics Letters 30(17), 2239-2241 (2005).

23 G. Delyon, F. Galland, and P. Réfrégier, "Minimal stochastic complexity image partitioning with unknown noise model," IEEE Transactions on image processing 15(10), 3207-3212 (2006).

24 J. W. Goodman, Statistical Optics, Wiley (2000).

25 M. Laurenzis, Y. Lutz, F. Christnacher, et al., "Homogeneous and speckle-free laser illumination for range-gated imaging and active polarimetry," Optical Engineering 51(6), 061302 (2012).

26 S. Covo and A. Elalouf, "A novel single-gamma approximation to the sum of independant gamma variables, and a generalization to infinitely divisible distributions," Electronic Journal of Statistics 8, 894-926 (2014). 
27 T. Stewart, L. Strijbosch, J. Moors, et al., "A simple approximation to the convolution of gamma distributions," Discussion Paper 2007-70, Tilburg University, Center for Economic Research (2007).

28 M. Nakagami, "The m-distribution-a general formula of intensity distribution of rapid fading," Statistical Methods in Radio Wave Propagation 2(9), 3-36 (1960).

29 J. M. Schmitt, S. H. Xiang, and K. M. Yung, "Speckle in Optical Coherence Tomography," Journal of Biomedical Optics 4(1), 95-105 (1999).

30 N. D. Lai, M. Brunel, and F. Bretenaker, "Green two-frequency pulsed laser : intracavity doubling of helicoidal eigenstates," Journal Optical Society of America B 20(4), 662-669 (2003).

31 G. Pillet, L. Morvan, D. Dolfi, et al., "Wideband dual-frequency lidar-radar for simultaneous velocity and high-resolution range profile measurements," Proceedings of SPIE 7323, 73230Z-73230Z-11 (2009).

32 J. Thévenin, M. Vallet, M. Brunel, et al., "Beat-note locking in dual-polarization lasers submitted to frequency-shifted optical feedback," Journal of the Optical Society of America B 28(5), 1104-1110 (2011).

33 G. Pillet, L. Morvan, M. Brunel, et al., "Dual-frequency laser at $1.5 \mu \mathrm{m}$ for optical distribution and generation of high-purity microwave signals," Journal of Lightwave Technology 26(15), 2764-2773 (2008).

No biographies available. 